\title{
Arresting the Catalytic Arginine in Chlorite Dismutases: Impact on Heme Coordination, Thermal Stability, and Catalysis
}

\author{
Daniel Schmidt, ${ }^{\perp}$ Ilenia Serra, ${ }^{\perp}$ Georg Mlynek, Vera Pfanzagl, Stefan Hofbauer, Paul G. Furtmüller, \\ Kristina Djinovic-Carugo, Sabine Van Doorslaer,* and Christian Obinger*
}

Cite This: Biochemistry 2021, 60, 621-634

Read Online

\section{ACCESS | Llll Metrics \& More | 回 Article Recommendations | (s) Supporting Information}

ABSTRACT: Chlorite dismutases (Clds) are heme $b$-containing oxidoreductases that can decompose chlorite to chloride and molecular oxygen. They are divided in two clades that differ in oligomerization, subunit architecture, and the hydrogen-bonding network of the distal catalytic arginine, which is proposed to switch between two conformations during turnover. To understand the impact of the conformational dynamics of this basic amino acid on heme coordination, structure, and catalysis, Cld from Cyanothece sp. PCC7425 was used as a model enzyme. As typical for a clade $2 \mathrm{Cld}$, its distal arginine 127 is hydrogen-bonded to glutamine 74 . The latter has been exchanged with either glutamate (Q74E) to arrest R127 in a salt bridge or valine (Q74V) that mirrors the setting in clade $1 \mathrm{Clds}$. We present the X-ray crystal structures of Q74V and Q74E and demonstrate the $\mathrm{pH}$-induced changes in the environment and coordination of the heme

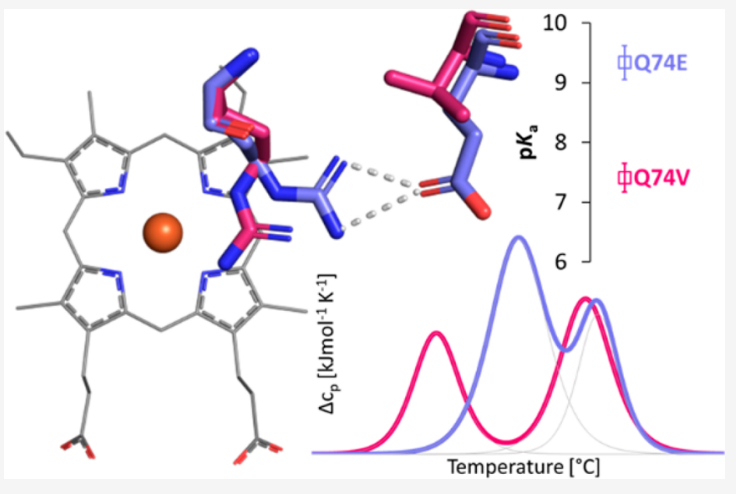
iron by ultraviolet-visible, circular dichroism, and electron paramagnetic resonance spectroscopies as well as differential scanning calorimetry. The conformational dynamics of R127 is shown to have a significant role in heme coordination during the alkaline transition and in the thermal stability of the heme cavity, whereas its impact on the catalytic efficiency of chlorite degradation is relatively small. The findings are discussed with respect to (i) the flexible loop connecting the $\mathrm{N}$-terminal and $\mathrm{C}$-terminal ferredoxin-like domains, which differs in clade 1 and clade 2 Clds and carries Q74 in clade 2 proteins, and (ii) the proposed role(s) of the arginine in catalysis.

$\mathrm{C}$ hlorite dismutases (Clds, EC 1.13.11.49) are heme bcontaining oxidoreductases found exclusively in prokaryotic organisms. ${ }^{1,2}$ They belong to the structural superfamily of porphyrin-binding dimeric $\alpha+\beta$ barrel proteins that typically show a high degree of functional diversity. ${ }^{3,4}$ Clds convert chlorite $\left(\mathrm{ClO}_{2}^{-}\right)$to chloride $\left(\mathrm{Cl}^{-}\right)$and dioxygen, therefore being of interest for biotechnological and bioremediation applications. ${ }^{1,2}$ The formation of an oxygen-oxygen bond during turnover constitutes an unusual biochemical reaction that otherwise is performed by only the water-splitting manganese complex of photosystem II in oxygenic organisms and an enzyme from an anaerobic methane-oxidizing bacterium. $^{5}$

Chlorite dismutases are divided into two lineages denoted as clade 1 ("long Clds") and clade 2 ("short Clds"). Figure 1 shows the X-ray crystal structures of two representative chlorite dismutases, namely, of the clade 1 Cld from Candidatus "Nitrospira defluvii" [NdCld, PDB entry 3NN1 (Figure 1)] and of the clade 2 Cld from Cyanothece sp. PCC7425 [CCld, PDB entry 5MAU (Figure 1)], respectively. The two clades exhibit differences in (i) oligomerization, with clade 1 proteins being homopenta- or homohexamers ${ }^{6-10}$ and clade 2 representatives forming homodimers, ${ }^{1-14}$ and (ii) subunit structure, with "long" clade 1 Clds being composed of an $\mathrm{N}$-terminal and a $\mathrm{C}$-terminal heme $b$-carrying ferredoxinlike domain and "short" clade 2 proteins lacking the $\alpha$-helices of the N-terminal domain (Figure 1). The C-terminal domain of clade 2 subunits is very similar to that of clade 1 enzymes, including the architecture of the heme cavity (Figure 1). 4,6,8,11,14 This high degree of similarity is reflected by similar redox properties and chlorite degradation activities. $1,2,15$

In the ferric resting state, the heme iron of Clds is ligated by a proximal histidine (H160 in NdCld and H114 in CCld) and a distal water. ${ }^{14}$ The only charged amino acid in the hydrophobic distal heme cavity is a fully conserved arginine (R173 in NdCld and R127 in CCld), which is proposed to be flexible and to switch between two conformations during catalysis, i.e., pointing toward either the heme iron ("in") or the substrate entry channel ("out")., $4,8,11,14,16$ Importantly, the hydrogen-bonding network of this catalytic arginine is different

Received: November 20, 2020

Revised: February 7, 2021

Published: February 15, 2021 


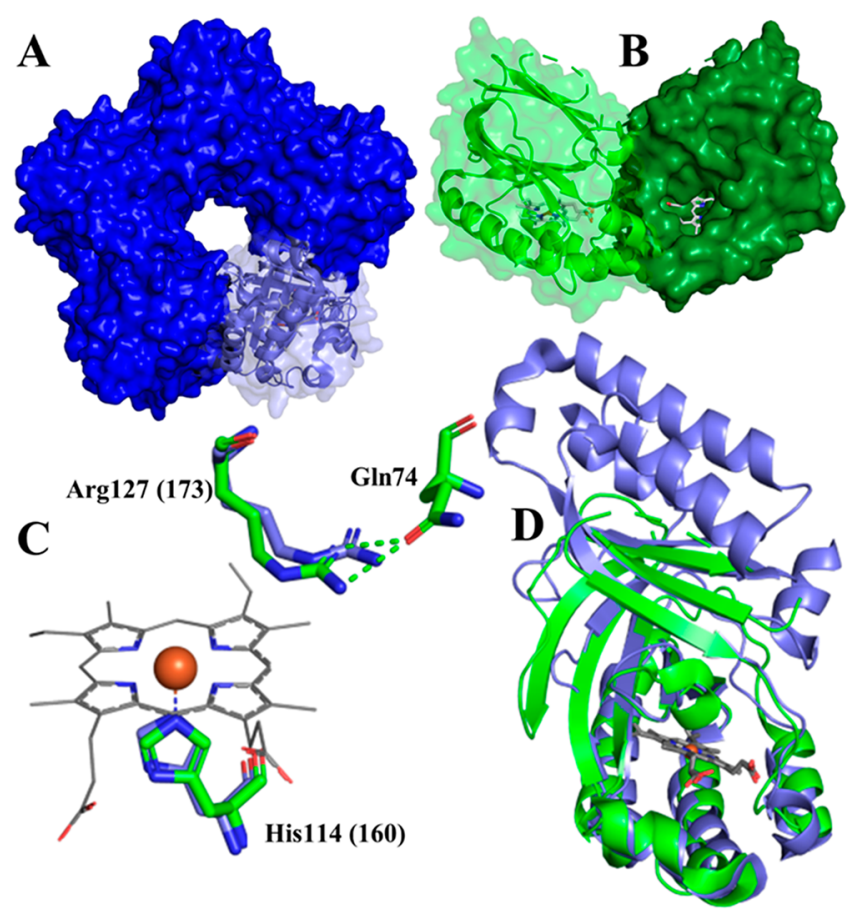

Figure 1. Structures of pentameric chlorite dismutase from N. defluvii $(\mathrm{NdCld}, \mathrm{PDB} \text { entry } 3 \mathrm{NN} 1)^{8}$ and dimeric chlorite dismutase from Cyanothece sp. PCC7425 (CCld, PDB entry 5MAU). ${ }^{14}$ (A) Surface representation of the $\mathrm{NdCld}$ pentamer. One subunit is depicted in ribbon representation, with a transparent surface illustration. (B) Dimeric CCld with one subunit shown in ribbon representation and a transparent surface illustration. Heme prosthetic groups are shown as gray sticks, and the metal ion is shown as an orange sphere. (C) Alignment of active site residues of CCld (green) and NdCld (blue), focusing on the catalytically important arginine (R127 and R173). Amino acids are numbered according to CCld and NdCld (brackets). The heme group (gray) is depicted in line representation. (D) Superimposition of subunits of CCld (green) and NdCld (blue) in ribbon representation. This figure was generated using PyMOL (http://www.pymol.org/).

in clade 1 and clade 2 Clds. In clade 2 Clds, R127 is H-bonded to Q74 located on a flexible $\alpha$-helical loop (Figure 2 and Table $\mathrm{S} 1$ ), which in general connects the N-terminal and C-terminal ferredoxin-like domains in porphyrin-binding $\alpha+\beta$ barrel proteins. ${ }^{4}$ By contrast, in clade 1 Clds the corresponding residue [Q123 in NdCld (Table S1)] turns away from R173 due to a different conformation of this loop (Figure 2, loop colored blue). As a consequence, a valine comes close to R173 in NdCld. ${ }^{9,11}$

The conserved arginine has been the center of numerous structural and functional studies for its putative involvement in substrate recognition and/or in preventing irreversible enzyme inactivation. ${ }^{9,16,17}$ It has been postulated that this basic residue supports the (either heterolytic or homolytic) cleavage of chlorite and the recombination reaction between the postulated transient intermediates (hypochlorite and Compound I or chlorine monoxide and Compound II $)^{1,2,14,16}$ and dictates the pronounced $\mathrm{pH}$ dependence of chlorite degradation. $^{17,18}$

In the crystal structure of CCld, in the resting state the heme iron participates in a distal $\mathrm{H}$-bonding network that includes the metal ion, two water molecules (W501 and W502), ${ }^{14} \mathrm{R} 127$ in the "out" conformation, and Q74. ${ }^{14}$ At alkaline $\mathrm{pH}$, deprotonation of W501 shortens its distance to the heme

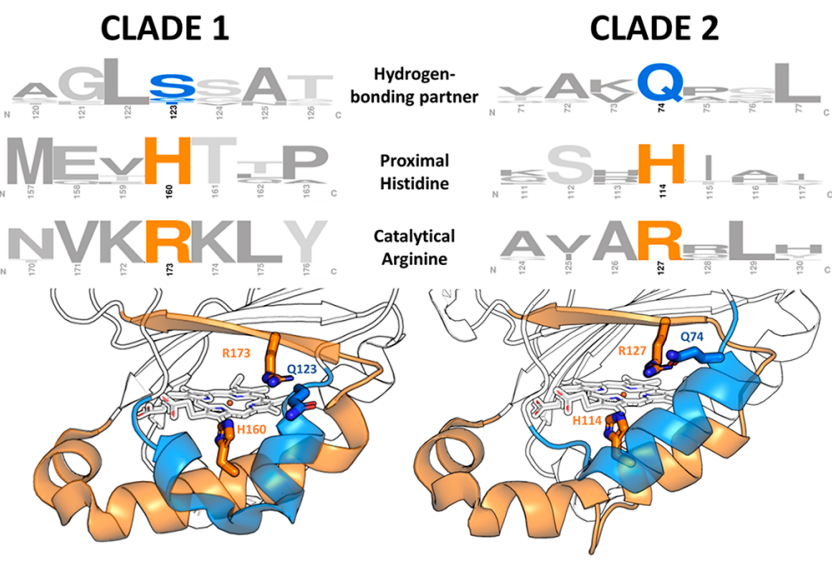

Figure 2. Comparison of the active site residues and architecture of clade 1 and clade 2 chlorite dismutases. Sequence alignments are represented as WebLogos. Structural differences are shown in cartoon representation, with highlighted amino acid residues depicted as sticks. The heme $b$-binding ferredoxin-like folds with catalytically important arginine (clade 1, R173; clade 2, R127) and proximal histidine (H160, H114) residues are colored orange. The linker region carrying the potential hydrogen-bonding partner of catalytic arginine (Q123 and Q74) is colored blue. This figure was generated using PyMOL (http://www.pymol.org/).

iron and significantly weakens or even breaks the interaction between $\mathrm{OH}^{-}$and W502 and simultaneously strengthens the interaction between W502 and R127. ${ }^{14}$ It was hypothesized that this alkaline transition might contribute to the pronounced $\mathrm{pH}$ dependence of chlorite degradation activity, which significantly decreases at alkaline $\mathrm{pH}$. ${ }^{1,2,7-9,13,14,17,18}$

Here, we aimed to study the impact of the flexibility and the H-bonding network of R127 in CCld by exchanging Q74 with valine $(\mathrm{Q} 74 \mathrm{~V})$ or glutamic acid (Q74E). The Q74V variant mirrors the situation in the clade 1 representative $\mathrm{NdCld}$ and should exhibit an increased flexibility of R127 compared to that of wild-type CCld, whereas Q74E might arrest the catalytic amino acid by forming a salt bridge. We provide the crystal structures of Q74V and Q74E and demonstrate the impact of the mutations and $\mathrm{pH}$ on heme coordination and active site architecture by ultraviolet-visible (UV-vis), electron paramagnetic resonance (EPR), and electronic circular dichroism (ECD) spectroscopy. Besides heme coordination, the mutations are shown to have a strong impact on the thermal stability of the heme cavity but an only weak effect on the chlorite degradation activity. The findings are discussed with respect to the proposed reaction mechanism(s) of the conversion of chlorite to chloride and dioxygen.

\section{MATERIALS AND METHODS}

Sequence Alignment. Multiple-sequence alignments of clade 1 and 2 Clds were constructed using ClustalW ${ }^{19}$ (Table S1) with the following parameters: gap opening penalty of 10 and gap extension penalties of 0.20 . All tools for sequence alignments were embedded in the MEGA X package. ${ }^{20}$ From these sequence alignments, sequence motifs were generated using WebLogo (http://weblogo.berkeley.edu/).

Cloning, Site-Directed Mutagenesis, Expression, and Purification. To generate plasmids for protein expression, the existing plasmid (pET-52 $\mathrm{b}^{+}$vector) encoding the $\mathrm{N}$-terminal HRV 3C-cleavable Strep(II)-tagged wild-type CCld was used 
as a template. ${ }^{13,14}$ As a result of low-purification yields using the existing Strep(II) tag, the affinity tag was exchanged with a His tag using polymerase chain reaction (primers listed in Table S2) and NEBuilder HiFi DNA Assembly (New England Biolabs, Ipswich, MA). To confirm successful cloning, the final plasmid was sequenced from primer pET-up (Microsynth, Balgach, Switzerland). CCld variants Q74E and Q74V were obtained by site-directed mutagenesis using the QuikChange Lightning Kit (Agilent, Santa Clara, CA). The modified wildtype plasmid described above was used as a template (Table S2).

Recombinant protein expression of wild-type CCld and variants was performed in Escherichia coli BL21 Gold (DE3) cells (Agilent) in LB medium supplemented with ampicillin. The cells were incubated at $37^{\circ} \mathrm{C}(180 \mathrm{rpm})$ until an $\mathrm{OD}_{600}$ of approximately 0.8 was reached. After the temperature had been decreased to $19^{\circ} \mathrm{C}$, protein expression was induced by adding $0.5 \mathrm{mM}$ isopropyl $\beta$-D-thiogalactopyranoside and the cultures were kept at this temperature overnight. The cells were harvested by centrifugation $\left(4^{\circ} \mathrm{C}, 5000 \mathrm{~g}, 20 \mathrm{~min}\right)$ and stored at $-30{ }^{\circ} \mathrm{C}$ until further purification.

For protein purification, the cell pellet was thawed and resuspended in lysis buffer [ $50 \mathrm{mM}$ phosphate buffer ( $\mathrm{pH} 7.4)$, $500 \mathrm{mM} \mathrm{NaCl}, 0.5 \%$ Triton X-100, and 5\% glycerol] with $\sim 100 \mu \mathrm{M}$ hemin. Following two $3 \mathrm{~min}$ cycles of ultrasonication (pulsed mode, $1 \mathrm{~s}$ sonication, $1 \mathrm{~s}$ rest, $90 \%$ ) on ice, the lysate was centrifuged $\left(4{ }^{\circ} \mathrm{C}, 17000 \mathrm{~g}, 35 \mathrm{~min}\right)$. The resulting supernatant was filtered $(0.45 \mu \mathrm{m}$, Durapore Membrane, Merck, Darmstadt, Germany) before being loaded onto a His-trap affinity column $(5 \mathrm{~mL}$; GE Healthcare, Chicago, IL) pre-equilibrated with binding buffer $[50 \mathrm{mM}$ phosphate buffer ( $\mathrm{pH} \mathrm{7.4)}$ and $500 \mathrm{mM} \mathrm{NaCl}$. After the loaded column had been washed with binding buffer, oncolumn cleavage of the His tag was performed by equilibrating with cleavage buffer $(50 \mathrm{mM}$ Tris- $\mathrm{HCl}$ with $150 \mathrm{mM} \mathrm{NaCl}$ and 1 mM EDTA) and cleaving with a His-tagged HRV 3C PreScission Protease overnight at $4{ }^{\circ} \mathrm{C}$. Elution was carried out with storage buffer $[50 \mathrm{mM}$ phosphate buffer $(\mathrm{pH} 7.0)]$ accompanied by a concentration and desalting step using an Amicon Ultra-15 centrifugal filter unit $(10 \mathrm{kDa}$ molecular weight cutoff; Merck). As a final polishing step, the concentrated protein was applied to a pre-equilibrated [50 $\mathrm{mM}$ phosphate buffer ( $\mathrm{pH} 7.0$ )] HiLoad 16/60 Superdex 200 prep grade column (GE Healthcare). The collected fractions were pooled, concentrated to $\sim 20 \mathrm{mg} \mathrm{mL}^{-1}$ using a centrifugal filter unit, and stored at $-80{ }^{\circ} \mathrm{C}$ in $50 \mu \mathrm{L}$ aliquots. Protein expression and purification of $\mathrm{NdCld}$ were performed as reported previously. ${ }^{9}$

UV-vis and ECD Spectroscopy. UV-vis spectra in a wavelength range between 200 and $700 \mathrm{~nm}$ were recorded routinely using a Cary $60 \mathrm{UV}-$ vis spectrophotometer (Agilent) and a model U-3900 spectrophotometer (Hitachi, Mannheim, Germany) at $25{ }^{\circ} \mathrm{C}$. The molar extinction coefficient of heme $\left(\varepsilon_{\text {Soret }}=100000 \mathrm{M}^{-1} \mathrm{~cm}^{-1}\right)$ was used to determine the enzyme concentration. To determine the $\mathrm{p} K_{\mathrm{a}}$ for the alkaline transition, $200 \mu \mathrm{L}$ of a $10 \mu \mathrm{M}$ enzyme solution in 5 $\mathrm{mM}$ sodium phosphate buffer $(\mathrm{pH} 7.0)$ was mixed with the same volume of $100 \mathrm{mM}$ buffer at the desired $\mathrm{pH}$ value (citrate-phosphate buffer, $\mathrm{pH} 4-6$; phosphate buffer, $\mathrm{pH}$ 6-9; borate-phosphate buffer, $\mathrm{pH} 9-12$ ). Absorption values at specific wavelengths reflecting peaks in difference spectra at acidic and alkaline $\mathrm{pH}$ regimes (i.e., 404, 406, 415, 423, 456, 508,575 , and $641 \mathrm{~nm}$ ) were plotted against $\mathrm{pH}$ values.
Sigmaplot (version 13.0, Systat Software, San Jose, CA) was used to fit the resulting curve to the following sigmoidal equation with $x_{0}$ corresponding to $\mathrm{p} K_{a} x$ being the $\mathrm{pH}$ of the buffer, and $y$ being the absorption at the observed wavelength:

$$
y=y_{0}+\frac{a}{1+\mathrm{e}^{-\left(\frac{x-x_{0}}{b}\right)}}
$$

Electronic circular dichroism spectroscopy was performed using Chirascan (Applied Photophysics, Leatherhead, U.K.). Spectra were recorded in the far-UV region $(180-260 \mathrm{~nm})$ and in the visible region $(260-500 \mathrm{~nm})$ using $10 \mu \mathrm{M} \mathrm{CCld}$. The path lengths for the far-UV and visible region were 1 and $10 \mathrm{~mm}$, respectively. Used buffers were $5 \mathrm{mM}$ citratephosphate buffer ( $\mathrm{pH} 5.0), 5 \mathrm{mM}$ phosphate buffer $(\mathrm{pH}$ 7.0), and $5 \mathrm{mM}$ borate-phosphate buffer ( $\mathrm{pH} 9.0$ ). The spectral bandwidth was set to $1 \mathrm{~mm}$, and the scan speed was 5 $\mathrm{s} \mathrm{nm}^{-1}$.

Temperature-mediated unfolding between 30 and $70{ }^{\circ} \mathrm{C}$ was monitored using a heat rate of $1{ }^{\circ} \mathrm{C} \mathrm{min}^{-1}$. Single-wavelength scans were performed in the far-UV $(210 \mathrm{~nm})$ and visible regions (Soret maximum). For data processing, Pro-Data Viewer provided by Applied Photophysics was used. The fraction of unfolded protein $(\alpha)$ was calculated according to the formula $\alpha=\left(\theta_{\mathrm{N}}-\theta\right) /\left(\theta_{\mathrm{N}}-\theta_{\mathrm{U}}\right)$, with $\theta$ being the ellipticity of the protein at a distinct temperature $(T), \theta_{\mathrm{N}}$ being the ellipticity (in millidegrees) at $210 \mathrm{~nm}$ or the Soret maximum (between 411 and $422 \mathrm{~nm}$, depending on the $\mathrm{pH}$ value) of the native protein, and $\theta_{\mathrm{U}}$ representing the ellipticity at $210 \mathrm{~nm}$ or the Soret maximum of the unfolded protein.

Electron Paramagnetic Resonance Spectroscopy. Continuous-wave (CW) EPR spectroscopy measurements were performed to assess the spin state of ferric wild-type forms of NdCld and CCld as well as the Q74V and Q74E variants and to investigate $\mathrm{pH}$-induced changes in the local environment of the heme iron. To maintain the consistency with other spectroscopic and biochemical assays, the EPR samples of $\mathrm{NdCld}$ and CCld were prepared in the same (or most similar) buffers used for the other experiments described in this work. Samples of wild-type NdCld were prepared at a concentration of $400 \mu \mathrm{M}$ in $50 \mathrm{mM}$ HEPES buffer ( $\mathrm{pH} 7.0$ ), in $50 \mathrm{mM}$ MES buffer ( $\mathrm{pH} 5.5)$, and in $50 \mathrm{mM}$ borate buffer $(\mathrm{pH}$ 10.0). Solutions of wild-type CCld, Q74V, and Q74E were prepared in a $50 \mathrm{mM}$ sodium phosphate-citrate buffer mixture ( $\mathrm{pH}$ 5.0), $50 \mathrm{mM}$ sodium phosphate (sodium phosphate monobasic-dibasic mixture) ( $\mathrm{pH} 7.0), 50 \mathrm{mM}$ sodiumphosphate-borate buffer mixture ( $\mathrm{pH} 9.0)$, and $50 \mathrm{mM}$ borate buffer $(\mathrm{pH} 10.0)$ at a concentration of $\sim 400 \mu \mathrm{M}$. To check the effect of a potential $\mathrm{pH}$ change upon freezing on EPR signatures, the EPR spectra of wild-type NdCld and CCld at $\mathrm{pH} 7$ were also recorded in a "temperature-independent" buffer (45\% HEPES and 55\% sodium phosphate ${ }^{21}$ ), and no significant changes were observed (data not shown). The influence of the cryoprotectant glycerol on the EPR spectra of wild-type NdCld and CCld was tested at neutral $\mathrm{pH}$. In the quartz EPR tubes (diameter of $4 \mathrm{~mm}$ ) containing the samples, the excess of paramagnetic $\mathrm{O}_{2}$ was removed in several freezepump-thaw cycles prior to the starting of the EPR experiment and all tubes were vacuum-pumped during the measurements.

$\mathrm{X}$-Band CW-EPR experiments were performed on a Bruker ESP300E spectrometer equipped with a liquid helium cryostat (Oxford Inc.) and operating at a microwave frequency of $\sim 9.44 \mathrm{GHz}$. Spectra were recorded at $10 \mathrm{~K}$, under nonsaturating conditions at microwave powers in the range of 2-5 

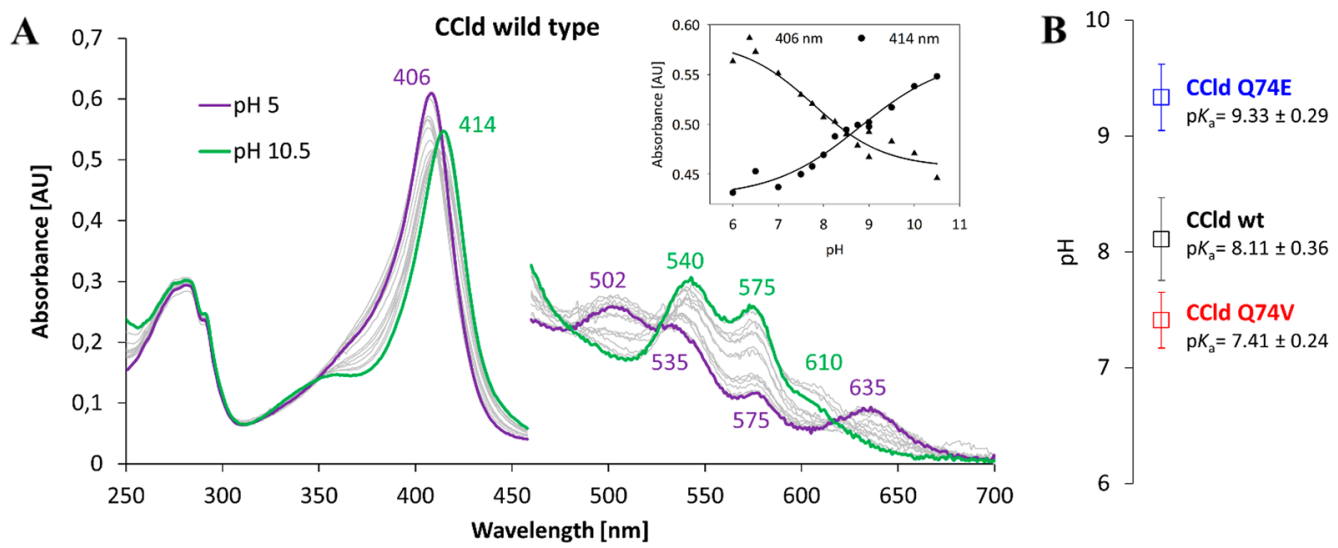

Figure 3. pH-dependent UV-vis spectral changes of wild-type CCld and variants Q74V and Q74E. (A) UV-vis spectra of wild-type CCld in the $\mathrm{pH}$ range of 5.0-10.5. The enzyme concentration was $5 \mu \mathrm{M}$. Very similar spectra were obtained for both variants (Figure S2). For better visualization of the CT bands and Q-bands, the spectra in the wavelength range between 460 and $700 \mathrm{~nm}$ are magnified 6-fold. The inset shows an increase and a decrease in absorbance at 406 and $414 \mathrm{~nm}$, respectively, with a change in $\mathrm{pH}$. The sigmoidal fit is shown as a black line. (B) Differences in $\mathrm{pK}_{\mathrm{a}}$ values of the alkaline transition between wild-type CCld and variants.

$\mathrm{mW}$, a modulation frequency of $100 \mathrm{kHz}$, and a modulation amplitude of $1 \mathrm{mT}$. Simulations of experimental spectra were performed with EasySpin software implemented in Matlab. ${ }^{22}$

Differential Scanning Calorimetry. Differential scanning calorimetry (DSC) experiments were performed on a MicroCal PEAQ-DSC Automated instrument (Malvern Panalytical Ltd., Malvern, U.K.) equipped with an autosampler for 96-well plates and controlled by the MicroCal PEAQ-DSC software (cell volume of $130 \mu \mathrm{L}$ ). Samples were analyzed over a temperature range of $20-80{ }^{\circ} \mathrm{C}$, using a heating scan rate of 60 ${ }^{\circ} \mathrm{C} \mathrm{h}^{-1}$. Each sample was immediately rescanned to check for reversible unfolding. Furthermore, if no refolding was detectable, this rescan was used as a baseline. CCld variants were applied as a $20 \mu \mathrm{M}$ solution in the range from $\mathrm{pH} 5.0$ to 9.0. For the $\mathrm{pH}$ dependence measurements of thermal unfolding, $50 \mathrm{mM}$ citrate phosphate buffer ( $\mathrm{pH}$ 5.0-6.0) and $50 \mathrm{mM}$ phosphate buffer ( $\mathrm{pH}$ 6.0-9.0) were used. Fitting of the data was performed with the MicroCal PEAQ-DSC software using a non-two-state equilibrium unfolding model.

X-ray Crystallography. Crystallization experiments were performed using the sitting drop vapor diffusion method in SWISSCI MRC three-well crystallization plates (Molecular Dimensions, Newmarket, U.K.). Crystallization drops were set up using a mosquito crystallization robot (TTP Labtech). The reservoir was filled with $40 \mu \mathrm{L}$ of a precipitant solution. In the sample wells, protein:precipitant ratios of 150:200, 200:200, and 250:200 were dispensed. The protein concentration was approximately $10 \mathrm{mg} / \mathrm{mL}$ in $50 \mathrm{mM}$ sodium phosphate buffer ( $\mathrm{pH}$ 7.0). In addition to known crystallization conditions [0.1 M MES ( $\mathrm{pH} 6.5$ ), $0.15 \mathrm{M} \mathrm{MgSO}_{4}, 28 \%(\mathrm{w} / \mathrm{v})$ polyethylene glycol 3350, and $3 \%(\mathrm{v} / \mathrm{v})$ glycerol]. ${ }^{14}$ Commercially available crystallization screens were used for further screening. Crystallization plates were stored in a Formulatrix RI-1000 imaging device at $22{ }^{\circ} \mathrm{C}$. Successful hits were obtained using the known conditions (Q74E) and the SG1 Screen from Molecular Dimensions (Q74V). Initial screening conditions were optimized for the growth of larger crystals by using the microseeding technique. For this method, a single crystal was placed in a crystallization solution in a fresh tube and crushed by vortexing using the Seed Bead kit (Hampton Research, Aliso Viejo, CA). The seeding solution was used in a ratio of 1:100 for further crystallization. Final conditions were as follows: $0.1 \mathrm{M}$ Tris ( $\mathrm{pH} 8.5$ ), $0.2 \mathrm{M} \mathrm{MgCl}_{2}$, and $20 \%(\mathrm{w} / \mathrm{v})$ PEG 8000 for Q74V and 0.1 M MES ( $\mathrm{pH} 6.5$ ), $0.15 \mathrm{M}$ $\mathrm{MgSO}_{4}, 28 \%(\mathrm{w} / \mathrm{v})$ PEG 3350, and 3\% (v/v) glycerol for Q74E. Crystals were soaked with mother liquor supplemented with $20 \%(\mathrm{w} / \mathrm{v})$ glycerol, harvested using a cryo-loop, and flash-vitrified in liquid nitrogen. Data were collected at $100 \mathrm{~K}$ using a PILATUS $6 \mathrm{M}$ detector $(25 \mathrm{~Hz}, 450 \mu \mathrm{m}$ sensor thickness) at beamline P13 operated by EMBL Hamburg at the PETRA III storage ring (DESY, Hamburg, Germany). ${ }^{23}$

Data sets were processed with XDS, and symmetry equivalent reflections merged with XDSCONV. ${ }^{24}$ Intensities were not converted to amplitudes. Initially, a conservative high-resolution cutoff $I / \sigma$ of $1-2$ was used. ${ }^{25}$ The phase problem was determined by molecular replacement using phenix.phaser ${ }^{26}$ taking the search model of PDB entry 5 MAU. ${ }^{14}$ The model was further improved by iterative cycles of manual model building using $\mathrm{COOT}^{27}$ and maximum likelihood refinement using phenix.refine. ${ }^{28}$ Phenix.refine converted intensities into amplitudes using the French and Wilson algorithm. ${ }^{29}$ The $B$-factor model was selected using the algorithm implemented in PDB_REDO, which is based on the number of X-ray reflections per atom and a recent implementation of the Hamilton test. ${ }^{30-32}$ The final highresolution cutoff was based on performing paired refinement using the PDB_REDO Web server. ${ }^{33}$ Final stages of refinement included either translation liberation screw (TLS) parameters, isotropic, or anisotropic B-factor models, automated addition of hydrogens and water molecules, optimization of X-ray/ADP weight, and optimization of X-ray/ stereochemistry weight. The model was validated with MolProbity. ${ }^{34}$ The figures were prepared with PyMOL Molecular Graphics System (version 2.3.4, Schrödinger, LLC). Atomic coordinates have been deposited in the Protein Data Bank as entries 7ATI (CCld Q74V) and 7ASB (CCld Q74E).

Chlorite Degradation Activity. Enzyme-mediated chlorite degradation was measured polarographically following the release of $\mathrm{O}_{2}$ by using a Clark-type oxygen electrode (Oxygraph Plus; Hansatech Instruments, Norfolk, U.K.). Reactions were monitored at $30{ }^{\circ} \mathrm{C}$ using a connected water bath. The electrode was calibrated by equilibrating to $100 \% \mathrm{O}_{2}$ saturation by bubbling with air and to $0 \% \mathrm{O}_{2}$ saturation by 
A
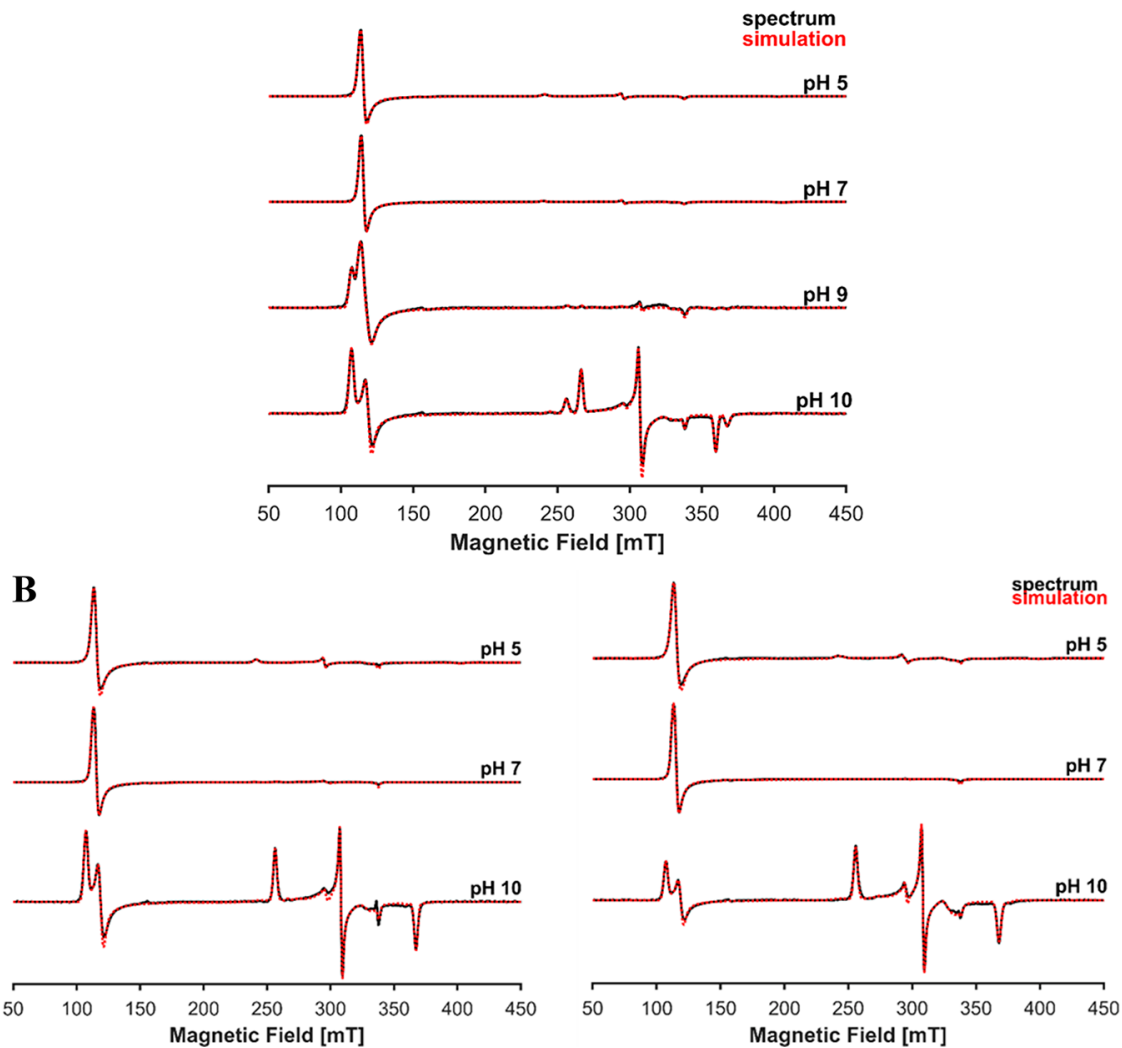

Figure 4. $\mathrm{pH}$ dependence of the CW-EPR spectrum of wild-type CCld and variants Q74V and Q74E. Samples were prepared in $50 \mathrm{mM}$ phosphatecitrate buffer ( $\mathrm{pH} 5.0$ ), $50 \mathrm{mM}$ phosphate buffer ( $\mathrm{pH} 7.0$ ), $50 \mathrm{mM}$ phosphate-borate buffer ( $\mathrm{pH} 9.0$ ), and $50 \mathrm{mM}$ borate buffer ( $\mathrm{pH} 10.0$ ). A microwave power of $2 \mathrm{~mW}$ was applied to record the spectra. Solid black for the experimental spectrum, dashed red for the simulation. (A) Wildtype CCld and (B) Q74E (left) and Q74V (right).

flushing with $\mathrm{N}_{2}$ until stable plateaus were reached to derive an offset and calibration factor. Reactions were performed in $\mathrm{O}_{2}$ free $50 \mathrm{mM}$ sodium phosphate buffer with $\mathrm{pH}$ values ranging from 5.0 to 9.0. The substrate was added to final concentrations from $20 \mu \mathrm{M}$ to $1 \mathrm{mM} \mathrm{NaClO}_{2}$. Concentrations of the chlorite stock solutions were measured using a molar extinction coefficient at $260 \mathrm{~nm}$ of $154 \mathrm{M}^{-1} \mathrm{~cm}^{-1}$.35,36 Subsequently, the reaction was started by adding $20 \mathrm{nM}$ CCld. Molecular oxygen production rates (micromolar $\mathrm{O}_{2}$ per second) were determined from the initial linear time traces and plotted against chlorite concentration. Furthermore, the total produced $\mathrm{O}_{2}$ (micromolar) was obtained by measuring the oxygen concentration until formation of a stable plateau after several minutes, depending on the CCld variant and the chlorite concentration.

Data Availability. The structures presented in this paper have all been deposited in the PDB as entries 7ASB (Q74E) and 7ATI $(\mathrm{Q} 74 \mathrm{~V})$. All remaining data are included herein.

\section{RESULTS}

Electronic Configuration of Ferric Wild-Type CCld and Variants Q74V and Q74E. The far-UV ECD spectra $(200-260 \mathrm{~nm})$ of wild-type CCld and the two variants are very similar (ellipticity minimum at $\sim 210 \mathrm{~nm}$ ), suggesting that the overall secondary structure has not been altered by the inserted mutations (Figure S1A). Increasing the $\mathrm{pH}$ from 5 to 9 had no impact on the spectral characteristics.
Both Q74V and Q74E show UV-vis spectral characteristics at acidic $\mathrm{pH}$ (i.e., $\mathrm{pH}$ 6.0) similar to those of the wild-type protein (Figure 3A, purple solid line; Figure S2). The ferric high-spin state is characterized by a Soret band at $406 \mathrm{~nm}, \mathrm{Q}$ bands at 502, 535, and $575 \mathrm{~nm}$, and a charge transfer band (CT) at $635 \mathrm{~nm}$. Under more alkaline conditions, a distinct shift to a low-spin form is observable. The Soret band is shifted to $414 \mathrm{~nm}$, and Q-bands at 540 and $575 \mathrm{~nm}$ and a weak CT band at $610 \mathrm{~nm}$ appear (Figure 3A, green solid line; Figure S2). These spectral properties are found in all CCld variants investigated in this study and are in agreement with previously reported data for the wild-type enzyme. ${ }^{13,14}$ Interestingly, while the spectral changes of the observed alkaline transition (i.e., deprotonation of distal water) are similar, the calculated $\mathrm{p} K_{\mathrm{a}}$ values differ significantly from that of wild-type CCld $\left(\mathrm{p} K_{\mathrm{a}}=\right.$ $8.11 \pm 0.36)$ with a $\mathrm{p} K_{\mathrm{a}}$ of $7.41 \pm 0.24$ for $\mathrm{Q} 74 \mathrm{~V}$ and $\mathrm{a} \mathrm{p} K_{\mathrm{a}}$ of $9.33 \pm 0.29$ for Q74E (Figure 3B).

Similar to the UV-vis experiments, the ECD spectra in the visible region strongly depend on $\mathrm{pH}$ (Figure S1B). The Soret bands exhibit a positive ellipticity with similar maxima at $\mathrm{pH}$ 5.0 (wild type, $414 \mathrm{~nm}$; Q74V, $413 \mathrm{~nm}$; Q74E, $412 \mathrm{~nm}$ ). With both wild-type CCld and Q74V, the Soret maxima are redshifted with an increase in $\mathrm{pH}(\mathrm{pH} \mathrm{7,415}$ and $416 \mathrm{~nm}$, respectively; $\mathrm{pH} 9,422$ and $423 \mathrm{~nm}$, respectively). By contrast, the shift is less pronounced in the Q74E variant ( $\mathrm{pH} 7.0,412$ $\mathrm{nm}$; $\mathrm{pH}$ 9.0, $415 \mathrm{~nm}$ ).

Next, the impact of the altered hydrogen-bonding network of distal arginine with particular attention to $\mathrm{pH}$-induced changes was probed by comprehensive EPR analysis and novel 
Table 1. EPR Simulation Parameters of Wild-Type CCld at Different $\mathrm{pH}^{\text {Values }}{ }^{a}$

\begin{tabular}{|c|c|c|c|c|c|c|c|c|c|}
\hline & species & $g_{x}^{\text {eff }}$ & $g_{y}^{\text {eff }}$ & $g_{z}^{\text {eff }}$ & $g_{x}$ & $g_{y}$ & $g_{z}$ & $E / D$ & $\%$ \\
\hline \multirow[t]{3}{*}{$\mathrm{pH} 5$} & HS1 & 5.92 & 5.84 & 2.00 & 1.96 & 1.96 & 2.00 & 0.002 & 53 \\
\hline & HS2 & 6.09 & 5.68 & 1.99 & 1.96 & 1.96 & 2.00 & 0.009 & 19 \\
\hline & LS1 & & & & 1.67 & 2.28 & 2.80 & - & 28 \\
\hline \multirow[t]{3}{*}{ pH 7} & HS1 & 5.90 & 5.86 & 2.00 & 1.96 & 1.96 & 2.00 & $<0.001$ & 63 \\
\hline & HS2 & 6.09 & 5.68 & 2.00 & 1.96 & 1.96 & 2.00 & 0.009 & 17 \\
\hline & LS1 & & & & 1.66 & 2.28 & 2.81 & - & 20 \\
\hline \multirow[t]{5}{*}{ pH 9} & HS1 & 5.90 & 5.86 & 2.00 & 1.96 & 1.96 & 2.00 & $<0.001$ & 13 \\
\hline & HS2 & 6.03 & 5.69 & 2.00 & 1.95 & 1.95 & 2.00 & 0.007 & 22 \\
\hline & HS3 & 6.28 & 5.65 & 1.99 & 1.99 & 1.99 & 2.00 & 0.013 & 47 \\
\hline & LS1-alkaline & & & & 1.88 & 2.26 & 2.53 & - & 7 \\
\hline & LS2-alkaline & & & & 1.83 & 2.19 & 2.63 & - & 11 \\
\hline \multirow[t]{4}{*}{$\mathrm{pH} 10$} & HS3 & 6.29 & 5.66 & 1.99 & 1.99 & 1.99 & 2.00 & 0.013 & 25 \\
\hline & LS1-alkaline & & & & 1.87 & 2.20 & 2.53 & - & 46 \\
\hline & LS2-alkaline & & & & 1.83 & 2.19 & 2.63 & - & 23 \\
\hline & LS3-alkaline & & & & 1.80 & 2.27 & 2.75 & - & 6 \\
\hline
\end{tabular}

${ }^{a}$ Errors in $g$ values of \pm 0.01 , errors in $E / D$ ratios of \pm 0.001 , and errors in contributions of $\pm 1 \%$.

Table 2. EPR Simulation Parameters of Variants Q74E and Q74V of CCld at Different pH Values ${ }^{a}$

\begin{tabular}{|c|c|c|c|c|c|c|c|c|c|}
\hline & species & $g_{x}^{\text {eff }}$ & $g_{y}^{\text {eff }}$ & $g_{z}^{\text {eff }}$ & $g_{x}$ & $g_{y}$ & $g_{z}$ & $E / D$ & $\%$ \\
\hline \multicolumn{10}{|l|}{ Q74E } \\
\hline \multirow[t]{3}{*}{$\mathrm{pH} 5$} & HS1 & 5.92 & 5.83 & 2.00 & 1.96 & 1.96 & 2.00 & 0.002 & 48 \\
\hline & HS2 & 6.14 & 5.69 & 2.00 & 1.97 & 1.97 & 2.00 & 0.010 & 22 \\
\hline & LS1 & & & & 1.67 & 2.28 & 2.79 & - & 30 \\
\hline \multirow[t]{5}{*}{$\mathrm{pH} 7$} & HS1 & 5.91 & 5.89 & 2.00 & 1.96 & 1.96 & 2.00 & 0.002 & 68 \\
\hline & HS2 & 6.16 & 5.68 & 2.00 & 1.97 & 1.97 & 2.00 & 0.010 & 13 \\
\hline & LS1 & & & & 1.66 & 2.28 & 2.81 & - & 7 \\
\hline & LS2 & & & & 1.81 & 2.27 & 2.62 & - & 8 \\
\hline & LS3 & & & & 1.89 & 2.27 & 2.45 & - & 4 \\
\hline \multirow[t]{4}{*}{$\mathrm{pH} 10$} & HS3 & 6.29 & 5.66 & 1.99 & 1.99 & 1.99 & 2.00 & 0.013 & 24 \\
\hline & LS1-alkaline & & & & 1.88 & 2.20 & 2.53 & - & 1 \\
\hline & LS2-alkaline & & & & 1.83 & 2.19 & 2.63 & - & 65 \\
\hline & LS3-alkaline & & & & 1.82 & 2.27 & 2.75 & - & 10 \\
\hline \multicolumn{10}{|l|}{ Q74V } \\
\hline \multirow[t]{4}{*}{ pH 5} & HS1 & 5.93 & 5.84 & 2.00 & 1.96 & 1.96 & 2.00 & 0.002 & 40 \\
\hline & HS2 & 6.15 & 5.68 & 1.99 & 1.97 & 1.97 & 2.00 & 0.010 & 23 \\
\hline & LS1 & & & & 1.68 & 2.30 & 2.79 & - & 29 \\
\hline & LS2 & & & & 1.89 & 2.28 & 2.72 & - & 8 \\
\hline \multirow[t]{3}{*}{$\mathrm{pH} 7$} & HS1 & 5.91 & 5.89 & 2.00 & 1.97 & 1.97 & 2.00 & $<0.001$ & 81 \\
\hline & HS2 & 6.16 & 5.68 & 2.00 & 1.97 & 1.97 & 2.00 & 0.010 & 16 \\
\hline & LS3 & & & & 1.89 & 2.27 & 2.45 & - & 3 \\
\hline \multirow[t]{3}{*}{$\mathrm{pH} 10$} & HS3 & 6.29 & 5.66 & 1.99 & 1.99 & 1.99 & 2.00 & 0.013 & 14 \\
\hline & LS2-alkaline & & & & 1.83 & 2.19 & 2.64 & - & 75 \\
\hline & LS3-alkaline & & & & 1.80 & 2.28 & 2.75 & - & 11 \\
\hline
\end{tabular}

${ }^{a}$ Errors in $g$ values of \pm 0.01 , errors in $E / D$ ratios of \pm 0.001 , errors in contributions of $\pm 1 \%$.

information about the amino acid environment at the heme site in clade 2 Clds is provided. The low-temperature CW-EPR spectra of $\mathrm{NdCld}$ and CCld at neutral $\mathrm{pH}$ have been reported previously. ${ }^{13,15,16}$

In high-spin $(S=5 / 2)$ ferric heme proteins, a very large zerofield splitting (ZFS) is responsible for the typical CW-EPR spectral shape observed at X-band. Because the ZFS is much larger than the photon energy of the microwaves used, only transitions in the first Kramers doublet $\left(m_{s}= \pm 1 / 2\right)$ can be detected. For this reason, EPR spectra of these proteins can be simulated either as effective $S=1 / 2$ systems, using a $\mathbf{g}^{\text {eff }}$ tensor, where $g_{x}{ }^{\text {eff }}$ and $g_{y}{ }^{\text {eff }}$ are symmetrically split at $\sim 6$ and $g_{z}{ }^{\text {eff }}$ is close to 2 , or as real $S=5 / 2$ systems, where the $g$ tensor is almost isotropic with principal values close to 2 , and the observed features depend essentially on the $E / D$ ratio between the rhombic $(E)$ and tetragonal $(D)$ zero-field splitting parameters. ${ }^{37}$ In this study, both simulation methods were used to obtain a complete picture of the systems under investigation. If a strong base is present at the sixth distal coordination position, the iron spin converts to the low-spin state $(S=1 / 2)$. In this case, the principal $g$ values can be taken as a fingerprint and can be compared with those of other hexacoordinated heme systems in an attempt to identify the nature of the distal ligand.

The EPR spectra of wild-type CCld at different $\mathrm{pH}$ values are shown in Figure 4A. In a manner consistent with the published results mentioned above, the spectrum of CCld at $\mathrm{pH} 7.0$ is composed of two components due to high-spin heme 
centers (HS1 and HS2) and a smaller low-spin contribution of uncertain origin (LS1) (EPR parameters used for the simulation are reported in Table 1). The most dominant spectral contribution (HS1) is characterized by a near-axial effective $\mathbf{g}$ tensor $\left(g_{x}{ }^{\text {eff }} \approx g_{y}{ }^{\text {eff }}\right)$ and concomitant very small $E / D$ value. Notably, in the case of $\mathrm{NdCld}$, the contributions due to high-spin hemes account for only $\sim 23 \%$ of the spectrum, which is instead dominated by a highly anisotropic low-spin signal (Table S3; simulations shown in Figure S3). No significant variations are observed when $\mathrm{CCld}$ is brought to acidic $\mathrm{pH}$ (Figure 4A, top), while a trend becomes evident when the $\mathrm{pH}$ is increased to 10 (Figure 4A, bottom). An alkaline transition is partially observed at $\mathrm{pH}$ 9.0, when the appearance of new low-spin features is accompanied by a change in the high-spin component, from axial to rhombic $\mathbf{g}^{\text {eff }}$ tensor. At $\mathrm{pH}$ 10.0, two main low-spin species, denoted as LS1alkaline and LS2-alkaline, are nicely resolved and the leftover high-spin ( 25\%) is found to be completely rhombic (HS3). The principal $g$ values of the low-spin components are typical of an $\mathrm{OH}^{-}$coordination of the heme iron at the sixth position on the distal site. ${ }^{12,38-40}$

At $\mathrm{pH} 7.0$, the EPR spectra of variants Q74E and Q74V largely resemble that of the wild-type protein (Figure 4 and Figure S4) and can be simulated with similar parameters (Table 2). In addition, Q74E shows the presence of small contributions from three different low-spin components, while in the spectrum of $\mathrm{Q} 74 \mathrm{~V}$, a single weak low-spin signal is barely observed (Figure S4). Remarkably, when the two proteins are measured at $\mathrm{pH} 5.0$ (Figure 4B, top), a contribution of the same low-spin species found in the wildtype protein is detected. In all three proteins, the spectral contribution of this species accounts for $\sim 30 \%$ of the total EPR spectrum. At $\mathrm{pH}$ 10.0, the dominant low-spin component in the variants has the same $g$ values as LS2-alkaline, while this was less abundant than LS1-alkaline in the wild-type case (Figure 4B and Figure S5). Furthermore, similar to wild-type CCld, an axial-to-rhombic conversion occurs for the high-spin state of both Q74E and Q74V when the $\mathrm{pH}$ is increased (Figure 4B). Overall, these data indicate that the site-directed mutation of the glutamine does not substantially perturb the spin state of the heme iron in the resting state of CCld. In addition, the EPR findings corroborate the $\mathrm{pH}$-dependent trend observed by UV-vis spectroscopy and resonance Raman spectroscopy of wild-type CCld. ${ }^{14}$

It is interesting to note that in the alkaline spectrum of $\mathrm{NdCld}$ (Figure S3) the major low-spin species (excluding that already present at neutral $\mathrm{pH}$ ) is characterized by $g$ values close to those of LS2-alkaline (Table S3). This similarity with the Q74 CCld variants is coherent with the absence of the conserved glutamine in $\mathrm{NdCld}$, which would suggest that the positioning of the arginine has a role in determining the binding mode of hydroxide.

Finally, the restricted flexibility of the arginine in wild-type CCld, compared to that of $\mathrm{NdCld}$, might also be responsible for the different behavior of the two proteins in the presence of glycerol, at least at neutral $\mathrm{pH}$. As depicted in Figure 5, the addition of $20 \%$ glycerol causes dramatic changes in the lowfield part of the spectrum of $\mathrm{NdCld}$ (contributions of high-spin species), which now reveals a species with increased rhombicity and a well-resolved axial component. On the contrary, the spectrum of CCld is scarcely affected by this compound, suggesting that the active site of this enzyme is less prone to accommodating the glycerol molecule. Preliminary

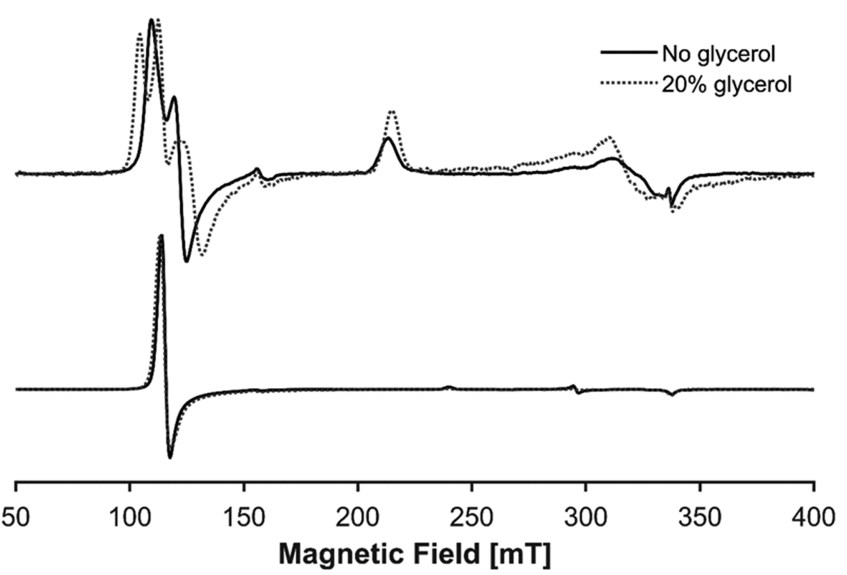

Figure 5. Effects of glycerol on the EPR spectra of wild-type $\mathrm{NdCld}$ and CCld at neutral $\mathrm{pH}$. Samples were prepared in $50 \mathrm{mM}$ HEPES buffer ( $\mathrm{pH} 7.0)$ (NdCld, top) and $50 \mathrm{mM}$ phosphate buffer ( $\mathrm{pH} 7.0$ ) (CCld, bottom). A microwave power of $5 \mathrm{~mW}$ was applied to record the spectra of $\mathrm{NdCld}$, while a microwave power of $2 \mathrm{~mW}$ was used for CCld spectra. Solid black for the sample without glycerol, dashed gray for the sample with $20 \%$ glycerol.

results on the effects of glycerol at higher $\mathrm{pH}$ values (data not shown) indicate a strong influence of $\mathrm{pH}$ in the reactivity of chlorite dismutases with this compound; however, this aspect will be explored more in detail in the future.

Thermal Stability of Wild-Type CCld and Variants Q74V and Q74E. Next, to investigate the influence of the introduced mutations and $\mathrm{pH}$ on protein stability, DSC and temperature-dependent ECD spectroscopy were performed. Figure 6 compares the thermograms of wild-type CCld and variants at $\mathrm{pH}$ 5.0. Two distinct endotherms were observed, suggesting a non-two-state transition $\left(T_{\mathrm{m}} 1\right.$ and $\left.T_{\mathrm{m}} 2\right)$ as described previously. ${ }^{41}$ In the $\mathrm{pH}$ regime of 5.0-9.0, the second transition $\left(T_{\mathrm{m}} 2\right)$ is at $\sim 58{ }^{\circ} \mathrm{C}$ for all three proteins, while significant differences were observed in $T_{\mathrm{m}} 1$ values, ranging from $38.3{ }^{\circ} \mathrm{C}(\mathrm{Q} 74 \mathrm{~V})$ to $50.9^{\circ} \mathrm{C}(\mathrm{Q} 74 \mathrm{E})$ (Figure 6). Both wild-type CCld and variants show the highest thermal stability at pH 6.0 (i.e., $T_{\mathrm{m}} 1=45.5-57.2{ }^{\circ} \mathrm{C}$; $T_{\mathrm{m}} 2=62.7-65.1$ ${ }^{\circ} \mathrm{C}$ ) and a continuously decreasing thermal stability at more alkaline $\mathrm{pH}$ (Figure 6).

The $\mathrm{pH}$ dependence of thermal stability was also investigated by ECD in the far- $\mathrm{UV}$ and visible region at $\mathrm{pH}$ 5.0, 7.0, and 9.0 to assign the observed transitions to distinct unfolding events. Similar to DSC, two transitions were observed with similar $T_{\mathrm{m}}$ values (Table S4). ECD in the visible region clearly correlates with the first unfolding event and heme release due to disappearance of Soret ellipticity (411-423 nm), whereas the second endotherm reflects melting of the secondary structures due to the loss of ellipticity at $210 \mathrm{~nm}$.

X-ray Crystal Structures of Variants Q74V and Q74E. The X-ray crystal structure of ferric wild-type CCld was already determined under various conditions, i.e., at $\mathrm{pH} 6.5$ and 8.5, and with soaked-in distal ligands $\left(\mathrm{F}^{-}\right.$and $\left.\mathrm{SCN}^{-}\right)$at $\mathrm{pH}$ 6.5. ${ }^{14}$ In all available CCld structures, the (putatively) flexible arginine (R127) is present in the "out" conformation, i.e., pointing toward the substrate entry channel. This conformation is stabilized by a hydrogen bond to glutamine 74 , which is in the proximity of $\mathrm{R} 127(2.85 \AA) .{ }^{14}$

Variants Q74V and Q74E crystallized in space groups $P 12_{1} 1$ and $P 1$, respectively, with one dimer per asymmetric unit. Each 

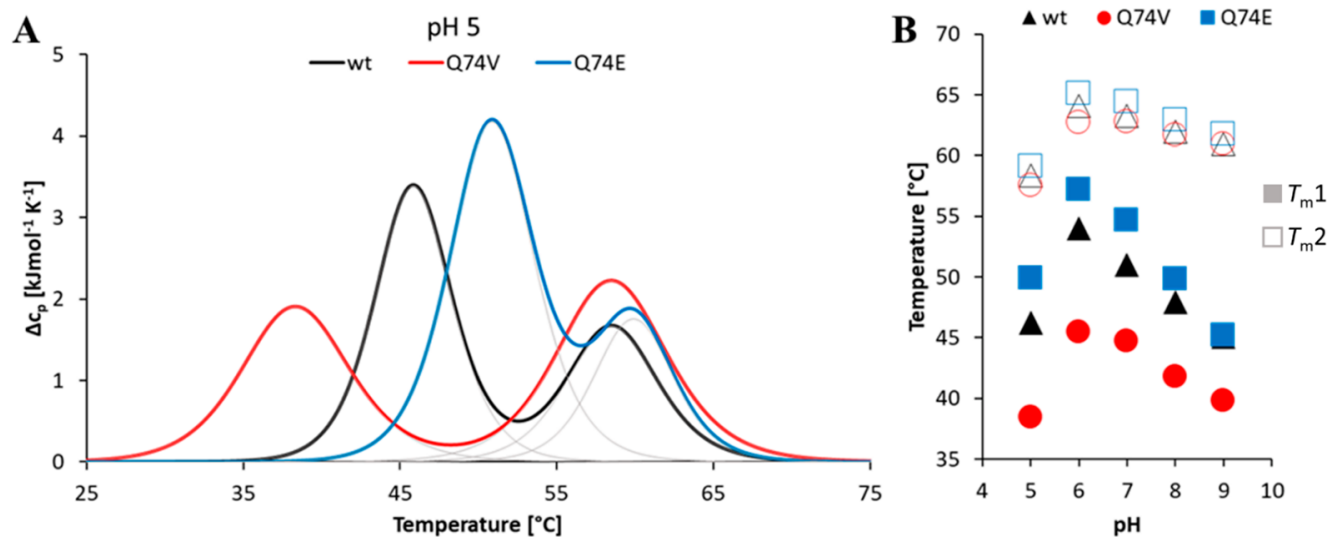

Figure 6. Thermal stability of wild-type CCld and variants measured at different $\mathrm{pH}$ values by differential scanning calorimetry. The enzyme concentration was $20 \mu \mathrm{M}$. (A) Normalized thermograms of wild-type CCld (black), Q74V (red), and Q74E (blue) at pH 5.0. Colored lines represent experimental data, and gray lines show a fit of the endotherm to a non-two-state transition model. (B) Calculated $T_{\mathrm{m}} 1$ (filled symbols) and $T_{\mathrm{m}} 2$ (empty symbols) plotted vs $\mathrm{pH}$ values. Measurements were performed in $50 \mathrm{mM}$ citrate phosphate buffer ( $\left.\mathrm{pH} 5.0-6.0\right)$ and $50 \mathrm{mM}$ phosphate buffer ( $\mathrm{pH} 7.0-9.0)$.

subunit of the homodimeric proteins binds one heme $b$. Similar to that of wild-type CCld ${ }^{14}$ the structure consists of a ferredoxin-like fold that is defined by five $\alpha$-helices along with a $\beta$-barrel composed of three-stranded and five-stranded antiparallel $\beta$-sheets. The crystals diffracted to high resolutions of $1.51 \AA(\mathrm{Q} 74 \mathrm{~V})$ at $\mathrm{pH} 8.5$ and $1.40 \AA(\mathrm{Q} 74 \mathrm{E})$ at $\mathrm{pH} 6.5$ (Table 3). Figure S6 shows a comparison of the $2 F_{\mathrm{o}}-F_{\mathrm{c}}$ electron density maps of the active sites of variants $Q 74 \mathrm{~V}$ and Q74E contoured at $\sigma=1.5$. The high degree of similarity with the overall structure of wild-type $\mathrm{CCld}^{14}$ is reflected by rootmean-square deviation (rmsd) values of $0.253 \AA$ (over $294 \mathrm{C}_{\alpha}$ atoms) and $0.055 \AA$ (over $330 \mathrm{C}_{\alpha}$ atoms) for Q74V and Q74E, respectively.

Histidine 114 serves as the fifth heme ligand on the proximal side, with the $\mathrm{N}_{\varepsilon} 2$ atom being $2.15 \AA$ from the heme iron in both structures (Figure 7B,D). On the distal side, a $\mathrm{H}_{2} \mathrm{O}$ molecule (W501) is situated above the heme iron at a distance of $2.6 \AA$. The conformation of the distal arginine was significantly affected by exchange of Gln74. While in Q74E (pH 6.5) R127 adopts a nearly identical ("out”) conformation as in wild-type CCld ${ }^{14}$ (Figure 7), in Q74V ( $\mathrm{pH} \mathrm{8.5)} \mathrm{it} \mathrm{is}$ pointing toward the heme iron (Figure 7). In Q74V (deprotonated), W501 is directly $\mathrm{H}$-bonded to $\mathrm{N}_{\varepsilon}(2.2 \AA)$ and $\mathrm{N}_{\eta} 2$ (2.3 $\AA$ ) of R127 (Figure 7). In Q74E, the distal W501 is hydrogen bonded to a second conserved water molecule (W502, $2.5 \AA$ ), which in turn forms an $\mathrm{H}$-bond to the $\mathrm{N}_{\varepsilon}$ atom $(2.0 \AA)$ of R127 in the "out" conformation, which is stabilized by a salt bridge between R127 and E74 (Figure 7). The distances between $\mathrm{N}_{\eta} 1$ and $\mathrm{N}_{\eta} 2$ of R127 and $\mathrm{O}_{\varepsilon} 1$ of E74 are 2.3 and $2.6 \AA$, respectively. The second available hydrogen of $\mathrm{N}_{\eta} 2$ is further H-bonded to a $\mathrm{H}_{2} \mathrm{O}$ molecule (W503, $2.0 \AA$ ), which in addition forms a hydrogen bond to W502. In Q74V, the "in" conformation of R127 leads to altered H-bonds of $\mathrm{N}_{\eta} 1$ $(2.5 \AA)$ and $\mathrm{N}_{\eta} 2(2.3 \AA)$ to W503. Furthermore, $\mathrm{N}_{\eta} 1$ of R127 is $\mathrm{H}$-bonded to an additional $\mathrm{H}_{2} \mathrm{O}$ molecule (W504), which is not present in wild-type $\mathrm{CCld}^{14}$ and Q74E (Figure 7B,D). Interestingly, the architecture of the $\alpha$-helical loop, which connects the $\mathrm{N}$-terminal and $\mathrm{C}$-terminal ferredoxin-like fold in CCld protomers, is almost identical in the three proteins.

Kinetics of Chlorite Degradation. Interestingly, the catalytic efficiency $\left(k_{\text {cat }} / K_{\mathrm{M}}\right)$ of chlorite degradation of both variants was similar to that of the wild-type protein (Table 4) with a $\mathrm{pH}$ optimum around 5 . In the $\mathrm{pH}$ regime from 5.0 to 9.0, Q74V exhibits the lowest $K_{\mathrm{M}}$ values, which decrease with an increase in $\mathrm{pH}(\mathrm{pH} 5.0,47.8 \mu \mathrm{M} ; \mathrm{pH}$ 9.0, $19.3 \mu \mathrm{M})$. In both wild-type CCld and Q74E, the same $\mathrm{pH}$ dependence of $K_{\mathrm{M}}$ values is observed [wild-type CCld, $127.1 \mu \mathrm{M}$ ( $\mathrm{pH} 5.0$ ) and 59.2 $\mu \mathrm{M}(\mathrm{pH} 9.0) ; \mathrm{Q} 74 \mathrm{E}, 327.0 \mu \mathrm{M}(\mathrm{pH} 5.0)$ and 21.0 $\mu \mathrm{M}$ ( $\mathrm{pH} 9.0)]$. With all three proteins, the $k_{\text {cat }}$ values are highest at acidic $\mathrm{pH}$ values and decrease with an increase in $\mathrm{pH}$ (Table 4). At the $\mathrm{pH}$ optimum, the $k_{\text {cat }}$ values decrease in the following order: Q74E > wild-type CCld > Q74V. It must be mentioned that above $100 \mu \mathrm{M}$ chlorite (5000-fold excess) irreversible inactivation is observed with all three proteins. At $\mathrm{pH}>7.0$, both $\mathrm{Q} 74 \mathrm{~V}$ and $\mathrm{Q} 74 \mathrm{E}$ are more prone to inactivation compared to wild-type CCld (Figure S7).

\section{DISCUSSION}

Functional Clds possess a conserved distal arginine in an otherwise hydrophobic active site pocket, which in all crystal structures of clade 2 Clds published thus far (including the model enzyme CCld) is in the "out" conformation and forms a hydrogen bond to Q74. Glutamine 74 is located on the $\alpha$ helical loop that connects the $\mathrm{N}$ - and $\mathrm{C}$-terminal domains of a protomer. ${ }^{1,14}$ In clade $1 \mathrm{Clds}$, a hydrogen-bonding partner is missing due to different conformations of this loop region (Figure 2). ${ }^{7-9}$ CCld variant $\mathrm{Q} 74 \mathrm{~V}$ was designed to mimic this setting in clade $1 \mathrm{Clds}$, whereas the design of CCld variant Q74E aimed to study the impact of arresting R127 in a salt bridge with E74.

In the crystal structure of Q74V determined at $\mathrm{pH} 8.5, \mathrm{R} 127$ adopts the "in" conformation and directly interacts with the deprotonated distal W501 (Figure 7), which is different to the orientation of R127 in X-ray and neutron structures of wildtype CCld determined at $\mathrm{pH} 6.5,8.5$, and $\mathrm{pH} 9.0$ as well as those of complex structures with fluoride and thiocyanate. ${ }^{14}$ In all of these wild-type CCld data sets, ${ }^{14}$ the R127 side chain is positioned away from the heme moiety. This "out" conformation is stabilized by hydrogen bonding between $\mathrm{N}_{\eta} 1$ of $\mathrm{R} 127$ and $\mathrm{O}_{\varepsilon} 1$ of $\mathrm{Q} 74 .{ }^{14}$ Because the $\mathrm{p} K_{\mathrm{a}}$ for the alkaline transition in $\mathrm{Q} 74 \mathrm{~V}$ has been determined to be 7.41 compared to 8.11 for wild-type CCld (Figure 3), it is reasonable to assume that its (more flexible) guanidinium group promoted the deprotonation of W501. Both the decrease in the $\mathrm{p} K_{\mathrm{a}}$ of 
Table 3. Crystallization Conditions and Data Collection and Refinement Statistics for CCld Variants Q74V (PDB entry 7ATI) and Q74E (PDB entry 7ASB) ${ }^{a}$

\begin{tabular}{|c|c|c|}
\hline & CCld Q74V & CCld Q74E \\
\hline wavelength $(\AA)$ & 0.9763 & 0.9763 \\
\hline $\begin{array}{l}\text { resolution range } \\
\text { (A) }\end{array}$ & $\begin{array}{l}29.64-1.51 \\
\quad(1.564-1.51)\end{array}$ & $30.98-1.4(1.45-1.4)$ \\
\hline space group & $P 12_{1} 1$ & $P 1$ \\
\hline $\begin{array}{l}\text { unit cell } \\
\text { dimensions }\end{array}$ & $\begin{array}{l}55.014 \AA, 72.676 \AA \text {, } \\
60.169 \AA, 90^{\circ}, \\
112.733^{\circ}, 90^{\circ}\end{array}$ & $\begin{array}{l}51.16 \AA ̊ ㇒, 52.738 \AA \\
54.772 \AA, 107.41^{\circ} \\
99.143^{\circ}, 108.984^{\circ}\end{array}$ \\
\hline $\begin{array}{l}\text { total no. of } \\
\text { reflections }\end{array}$ & 457965 (42914) & $325936(32812)$ \\
\hline $\begin{array}{l}\text { no. of unique } \\
\text { reflections }\end{array}$ & $68449(6796)$ & $90357(8896)$ \\
\hline multiplicity & $6.7(6.3)$ & $3.6(3.7)$ \\
\hline completeness (\%) & $98.28(84.44)$ & $92.53(91.39)$ \\
\hline mean $I / \sigma(I)$ & $11.45(0.35)$ & $10.09(1.14)$ \\
\hline $\begin{array}{l}\text { Wilson B-factor } \\
\quad\left(\AA^{2}\right)\end{array}$ & 25.32 & 17.09 \\
\hline$R_{\text {merge }}$ & $0.09328(4.026)$ & $0.06724(1.195)$ \\
\hline$R_{\text {meas }}$ & $0.1012(4.39)$ & $0.07945(1.396)$ \\
\hline$R_{\text {pim }}$ & $0.03886(1.721)$ & $0.04186(0.7172)$ \\
\hline$C C_{1 / 2}$ & $0.999(0.169)$ & $0.998(0.571)$ \\
\hline$C C^{*}$ & $1(0.537)$ & $0.999(0.852)$ \\
\hline $\begin{array}{l}\text { no. of reflections } \\
\text { used in } \\
\text { refinement }\end{array}$ & $67369(5762)$ & $90301(8887)$ \\
\hline $\begin{array}{l}\text { no. of reflections } \\
\text { used for } R_{\text {free }}\end{array}$ & $1575(132)$ & $1384(151)$ \\
\hline$R_{\text {work }}$ & $0.1738(0.4070)$ & $0.1637(0.3323)$ \\
\hline$R_{\text {free }}$ & $0.1956(0.3953)$ & $0.1825(0.3423)$ \\
\hline CC (work) & $0.972(0.489)$ & $0.966(0.804)$ \\
\hline CC (free) & $0.965(0.268)$ & $0.940(0.710)$ \\
\hline $\begin{array}{l}\text { no. of non- } \\
\text { hydrogen atoms }\end{array}$ & 3470 & 3673 \\
\hline macromolecules & 3065 & 3057 \\
\hline ligands & 136 & 173 \\
\hline solvent & 269 & 443 \\
\hline protein residues & 364 & 362 \\
\hline $\begin{array}{l}\text { rmsd for bonds } \\
(\AA)\end{array}$ & 0.01 & 0.023 \\
\hline $\begin{array}{l}\text { rmsd for angles } \\
\text { (deg) }\end{array}$ & 0.94 & 2.18 \\
\hline $\begin{array}{l}\text { Ramachandran } \\
\text { favored }(\%)\end{array}$ & 97.22 & 97.49 \\
\hline $\begin{array}{l}\text { Ramachandran } \\
\text { allowed (\%) }\end{array}$ & 2.78 & 2.23 \\
\hline $\begin{array}{l}\text { Ramachandran } \\
\text { outliers (\%) }\end{array}$ & 0 & 0.28 \\
\hline $\begin{array}{l}\text { Rotamer outliers } \\
(\%)\end{array}$ & 0 & 0 \\
\hline Clashscore & 1.41 & 6.69 \\
\hline $\begin{array}{l}\text { average } B \text {-factor } \\
\left(\AA^{2}\right)\end{array}$ & 35.7 & 25.31 \\
\hline macromolecules & 35.47 & 23.44 \\
\hline ligands & 37.56 & 30.4 \\
\hline solvent & 37.38 & 36.26 \\
\hline no. of TLS groups & 19 & \\
\hline
\end{tabular}

${ }^{a}$ Statistics for the highest-resolution shell are shown in parentheses.

the alkaline transition and the X-ray structure suggest a higher flexibility in solution of R127 in Q74V compared to the wildtype protein. The opposite trend is visible for variant Q74E. At $\mathrm{pH} 6.5$, its active site architecture strongly resembles that of wild-type CCld with the exception that R127 is arrested in a salt bridge with E74 (Figure 7). As a consequence, its

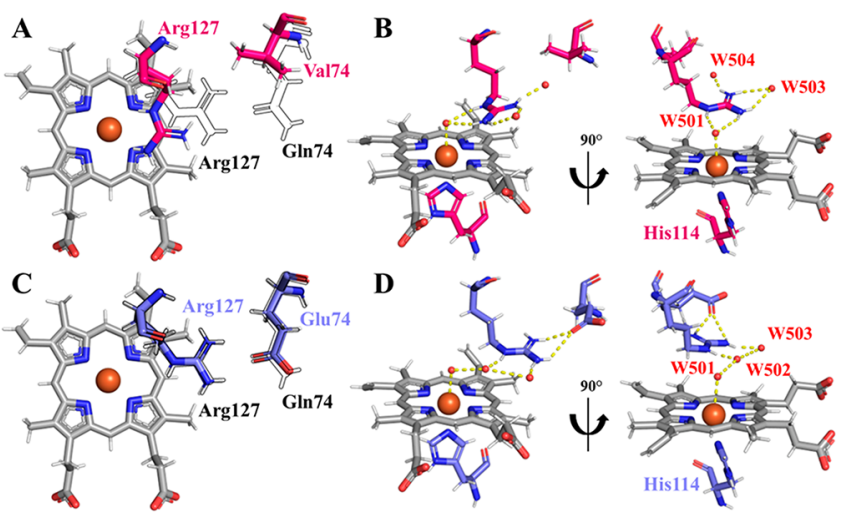

Figure 7. Active site architecture of CCld variants Q74V ( $\mathrm{pH} 8.5$ ) and Q74E ( $\mathrm{pH}$ 6.5). (A and C) Overlay of the important residues on the distal side (Arg127 and Q/V/E74) of the heme cofactor (gray) in stick representation, with the respective CCld wild-type residues (PDB entry 5MAU) shown as a black outline. (A) Q74V (red) shows a conformational change to the "in" conformation, while Q74E (C, blue) depicts an almost identical conformation compared to the wildtype structure. (B and D) Detailed view of the active site architecture in two different views. Heme iron and waters (W) are shown as orange and red spheres, respectively. Hydrogen bonds are depicted with a yellow dashed line. Highly conserved His114 serves as the fifth heme ligand on the proximal side. This figure was generated using PyMOL (http://www.pymol.org/).

Table 4. Kinetic Parameters of Wild-Type CCld and Variants Q74V and Q74E at $\mathrm{pH}$ 5.0, 7.0, and 9.0

$\begin{array}{cccc}\text { CCld } & K_{\mathrm{M}}(\mu \mathrm{M}) & k_{\text {cat }}\left(\mathrm{s}^{-1}\right) & k_{\text {cat }} / K_{\mathrm{M}}\left(\mathrm{M}^{-1} \mathrm{~s}^{-1}\right) \\ \mathrm{pH} 5 & & & \\ \quad \text { wild type } & 127.1 \pm 28.0 & 475.8 \pm 34.7 & (3.7 \pm 1.2) \times 10^{6} \\ \text { Q74V } & 47.8 \pm 4.7 & 280.2 \pm 2.8 & (5.9 \pm 0.7) \times 10^{6} \\ \text { Q74E } & 327.0 \pm 6.6 & 977.4 \pm 34.4 & (3.0 \pm 0.2) \times 10^{6} \\ \text { pH 7 } & & & \\ \quad \text { wild type } & 43.2 \pm 5.2 & 60.0 \pm 0.7 & (1.4 \pm 0.2) \times 10^{6} \\ \text { Q74V } & 18.8 \pm 3.5 & 50.8 \pm 0.4 & (2.7 \pm 0.5) \times 10^{6} \\ \text { Q74E } & 53.8 \pm 13.1 & 37.2 \pm 1.1 & (6.9 \pm 2.0) \times 10^{5} \\ \text { pH 9 } & & & \\ \text { wild type } & 59.2 \pm 20.1 & 33.4 \pm 1.5 & (5.6 \pm 2.5) \times 10^{5} \\ \text { Q74V } & 19.3 \pm 6.0 & 43.0 \pm 0.7 & (2.2 \pm 0.8) \times 10^{6} \\ \text { Q74E } & 21.0 \pm 11.2 & 18.91 \pm 0.5 & (0.9 \pm 0.7) \times 10^{6}\end{array}$

${ }^{a}$ Chlorite degradation was monitored polarographically.

guanidinium group is not able to stabilize the conjugated base of W501 and the $\mathrm{p} K_{\mathrm{a}}$ of the alkaline transition is significantly increased to a $\mathrm{p} K_{\mathrm{a}}$ of 9.33 (Figure 3). These findings clearly demonstrate experimentally that (i) the distal arginine in wildtype Clds is flexible, (ii) its dynamics is influenced by its remote $\mathrm{H}$-bonding partner, and (iii) in Q74V (mirroring clade 1 enzymes) this flexibility is more pronounced than in wildtype CCld.

Most interestingly, these differences in the conformational dynamics of R127 had a strong impact on the thermal stability of the heme cavity. In principle, the thermal stability of pentameric clade $1 \mathrm{Clds}$ (i.e., $\mathrm{NdCld}$ ) is significantly higher than that of dimeric clade 2 Clds like CCld. Thermal unfolding of clade 1 enzymes follows a simple two-state transition, suggesting a cooperative process, in which separation of subunits, monomer melting, and release of the heme cofactor occur simultaneously. ${ }^{41}$ By contrast, dimeric Clds, including CCld, follow a non-two-state unfolding pathway with two 
consecutive unfolding events. The differences in $\mathrm{N}$-terminal domain architecture and protomer interactions ${ }^{6-8,11,14}$ have been proposed to be responsible for these observations. ${ }^{38}$ As typical clade 2 Clds, wild-type CCld and variants Q74V and Q74E show two endotherms in DSC with the second unfolding event (i.e., melting of the overall secondary structures) occurring at similar $T_{\mathrm{m}} 2$ values $\left(\sim 58{ }^{\circ} \mathrm{C}\right)$ in the three proteins. By contrast, the first unfolding event strongly depends on the $\mathrm{H}$-bonding network and conformational dynamics of $\mathrm{R} 127$. At $\mathrm{pH} 5.0$, the $T_{\mathrm{m}} 1$ values increase in the following order: Q74V $\left(38.3{ }^{\circ} \mathrm{C}\right)<$ wild-type CCld $\left(45.8^{\circ} \mathrm{C}\right)$ $<$ Q74E $\left(50.9^{\circ} \mathrm{C}\right)$. As suggested by thermal ECD data in the visible region, at these temperatures the active site unfolds and heme is released (Table S4). In the case of Q74E, the salt bridge of R127 with E74, which is located at the $\alpha$-helical loop (Figures 2 and 7), stabilizes the heme cavity by increasing the conformational stability of the $\alpha$-helical loop (Figure 2). Disruption of the salt bridge at $50.9{ }^{\circ} \mathrm{C}(\mathrm{pH} 5.0)$ seems to initiate unfolding of the loop and opening of the heme cavity in Q74E.

At acidic and neutral $\mathrm{pH}$, the EPR spectra of the three proteins do not show significant differences (Figure 4). At $\mathrm{pH}$ 7.0, the high-spin ferric heme signal of Q74V still accounts for $97 \%$ of the spectrum, whereas the spectrum of Q74E already shows some minor low-spin signals (Figure S4). This may seem partially inconsistent with the $\mathrm{p} K_{\mathrm{a}}$ values obtained from UV-vis spectroscopy (Figure 3 and Figure S2); however, in EPR, the effects of freezing and formation of artifacts at cryogenic temperatures have to be taken into account. ${ }^{42,43}$ In any case, the spectroscopic findings clearly demonstrate that at the $\mathrm{pH}$ optimum of chlorite degradation activity (i.e., $\mathrm{pH} \mathrm{5)}$ (i) the heme coordination in the three proteins is similar, i.e., the geometry of the paramagnetic center is not substantially perturbed, and consequently (ii) the impact of the conformational dynamics of R127 is small.

In the alkaline $\mathrm{pH}$ regime, the geometries of the heme pockets of wild-type CCld and the variants Q74V and Q74E change. This includes changes in the rhombicity of the EPR signals of the high-spin components and formation of new lowspin species whose EPR features are consistent with the ones of hydroxo-ligated forms described for other chlorite dismutases. $^{12,38-40}$ The attribution of these signals to $\mathrm{OH}^{-}$ adducts is additionally supported by resonance Raman spectroscopy studies, which highlighted the presence of a $\mathrm{Fe}-\mathrm{OH}$ bond in the alkaline forms of several chlorite dismutases from both clade I and clade II. ${ }^{14,17,18,44}$ In wildtype CCld (Figure 4A), the high-spin components with an axial $\mathrm{g}^{\text {eff }}$ tensor, which dominate the spectrum at acidic and neutral $\mathrm{pH}$ (HS1 and HS2), progressively convert to a single and wellresolved rhombic species (HS3) at $\mathrm{pH} 10.0$ (Figure 4A). This reflects a different symmetry and altered geometry of the active site pocket most probably by repositioning of R127 and its Hbonding network. This is further corroborated by the analysis of the low-spin species identified at $\mathrm{pH} 10.0$ in both CCld and NdCld (Tables 1 and 2, Table S2, and Figures S3 and S5). LS1-alkaline $\left(g_{x}=1.87, g_{y}=2.20, g_{z}=2.53\right)$ and LS2-alkaline ( $\left.g_{x}=1.83, g_{y}=2.19, g_{z}=2.63\right)$ are the principal contributions in the high-pH spectrum of wild-type CCld, while a third species (LS3-alkaline: $g_{x}=1.80, g_{y}=2.27, g_{z}=2.75$ ) contributes to the spectrum to a lesser extent. These components have $g$ values similar to those of $\mathrm{OH}^{-}$adducts of other heme proteins, such as cytochrome $c$ peroxidase, ${ }^{45}$ human hemoglobin, ${ }^{46}$ horse heart myoglobin, ${ }^{46}$ and Caeno- rhabditis elegans globin-33. ${ }^{47}$ Nicoletti et al. showed that at high $\mathrm{pH}$ hemoglobin from Thermobifida fusca forms two hydroxo complexes with different sets of $g$ values, ${ }^{48}$ which they explained with the $\mathrm{OH}^{-}$group being hydrogen-bonded to different extents to nearby residues. In the case of hemoglobin, the component with broader features was assigned to a strong His $/ \mathrm{OH}^{-}$coordination. The differences in the H-bonding network most probably result in a different electron density distribution between the axial ligand(s) and the porphyrin, which primarily determines the magnitude of the tetragonal parameter according to crystal field theory. ${ }^{49,50}$ Indeed, as nicely exemplified by Svistunenko et al., ${ }^{46}$ the more unpaired electron density is on the heme iron, the more anisotropic is the $\mathbf{g}$ tensor of the low-spin signal. Therefore, it is possible that the LS contributions in the spectrum of wild-type CCld derive from the dynamics of R127, which allows the hydroxide group to attend different $\mathrm{H}$-bonding networks.

In contrast to wild-type CCld, the EPR spectrum at $\mathrm{pH} 10$ in both Q74E and Q74V is dominated by an LS2-alkaline contribution with larger $g$ anisotropy than the LS1-alkaline component, which suggests a higher spin density on the heme (Figure S5). This points to a different $\mathrm{H}$-bonding dynamics of the hydroxide group due to the altered flexibility of R127. Interestingly, the main hydroxide adduct in $\mathrm{NdCld}$ is characterized by $g$ values similar to those of LS2-alkaline (Figure S3), suggesting that the heme pocket of the pentameric Cld is very similar to that of CCld Q74V, which was designed as a model for clade 1 Clds.

The EPR spectra of both NdCld and CCld show features of low-spin states even at $\mathrm{pH} \leq 7$; however, their interpretation is not trivial. In the case of $\mathrm{NdCld}$, two broad low-spin contributions (Table S3, LS1' and LS2') have $g$ values consistent with other low-spin species previously observed in several EPR studies of chlorite dismutases at neutral $\mathrm{pH}$. While in certain cases their origin could not be determined, ${ }^{12}$ in the work of De Schutter et al. ${ }^{38}$ it was demonstrated that some of these species may represent "ready" states still accessible to the binding of external molecules. Moreover, in a comparative study of native and recombinant chlorite dismutase from Ideonella dechloratans, it was shown that a low-spin signal consistent with a bis-histidine coordination was present in only the recombinant form. ${ }^{51}$ In another work, the occurrence of the typical features of an imidazole adduct was explained as artifacts from the protein purification procedure. ${ }^{6}$ In the case of $\mathrm{NdCld}$, no imidazole was used during purification; therefore, this explanation can be ruled out. Furthermore, the predominance of the $\mathrm{LS1}^{\prime}$ signal in $\mathrm{NdCld}$ samples prepared at different $\mathrm{pH}$ values and in different buffers makes it unlikely to be due to the binding of an external molecule present in the medium and suggests a strong coordination from a ligand that cannot be easily displaced. For these reasons, the nature of the LS species remains elusive. In the case of CCld, the $g$ values of LS1 $\left(g_{x}=1.66, g_{y}=2.28, g_{z}=2.81\right)$ found at neutral and acidic $\mathrm{pH}$ are unique and (to the best of our knowledge) have never been reported before in Clds. This species is characterized by features that are consistent with neither imidazole-heme complexes ${ }^{6,12,52}$ nor the $\mathrm{OH}^{-}$adducts formed at high $\mathrm{pH}$, as shown above. However, these values present some similarities with the hydroxide complexes found in other heme protein samples at non-alkaline $\mathrm{pH} \cdot{ }^{43,46}$

Both UV-vis and EPR data have demonstrated that modulation of the H-bonding network of R127 in wild-type CCld and the two variants do not substantially perturb the spin 
state of the heme iron in the ferric resting state in solution in the acidic and neutral $\mathrm{pH}$ region. This fits with observation that at a $\mathrm{pH}$ optimum (i.e., $\mathrm{pH}$ 5.0) the catalytic efficiency of wild-type CCld and of the two variants is in the range of $3.0 \times$ $10^{6}$ to $5.9 \times 10^{6} \mathrm{M}^{-1} \mathrm{~s}^{-1}$, very similar to those of previously studied chlorite dismutases from clade 2 and most of clade 1 Clds (Table 4). ${ }^{8-14}$ Because even arresting R127 in a salt bridge (Figure 7) had almost no effect on the catalytic efficiency, this suggests that the impact of the conformational dynamics of $\mathrm{R} 127$ on catalysis is relatively small.

At the $\mathrm{pH}$ optimum and neutral $\mathrm{pH}$, the $K_{\mathrm{M}}$ values decrease in the following order: Q74E > wild-type CCld > Q74V. This mirrors the hierarchy of the thermal stability of the heme cavity. By contrast, the $k_{\text {cat }}$ values follow the opposite order at $\mathrm{pH}$ 5.0. On the basis of the findings presented here and recently published data on both clade 1 and clade 2 Clds, we propose the following functional role(s) of the only charged amino acid in the distal cavity of CCld.

The reaction cycle of dimeric CCld is initiated by binding of the anionic substrate $\left(\mathrm{p} K_{\mathrm{a}}=1.72\right)$ to the ferric heme center. At first sight, at the $\mathrm{pH}$ optimum in CCld the flexibility of the arginine seems to facilitate this reaction to some extent, because the $K_{\mathrm{M}}$ of Q74E was significantly higher compared to that of wild-type CCld. The differences in $K_{M}$ values are less pronounced at higher $\mathrm{pH}$ values. In pentameric $\mathrm{NdCld}$, it has been demonstrated that exchange of R173 with either alanine or glutamine had an only weak effect on the respective $K_{\mathrm{M}}$ values, suggesting that its role in chlorite binding is negligible.' This was further supported by MD data, which showed that R173 does not play a role in shuttling the anion into the active site. It rather followed the charged molecules and changes its orientation ("in" vs "out" accordingly), suggesting that its role in the initial reaction step is chlorite recognition only. ${ }^{53}$ By contrast, in clade 1 Cld from Dechloromonas aromatica exchange of R183 with alanine increased the $K_{\mathrm{M}}$ from 0.2 to $14.6 \mathrm{mM}^{17}$

Differences between clade 1 and clade 2 Clds may be related to the differences between the $\alpha$-helical loop that connects the $\mathrm{N}$ - and C-terminal domains of Cld protomers. Due to its location at the entrance to the heme cavity, it governs the accessibility to the heme cavity. In CCld variant Q74V and clade $1 \mathrm{CClds}$, the interaction between the distal arginine and the loop is small, whereas in wild-type CCld, a hydrogen bond between R127 and Q74 is formed. By contrast, Q74E increases the rigidity of the loop (as reflected by the DSC data) and thus decreases its dynamics, which seems to be important for chlorite accession and binding. Principally, chlorite binding should occur spontaneously and independent of arginine as demonstrated for $\mathrm{NdCld}$. This study also rules out that the alkaline transition is responsible for the $\mathrm{pH}$ dependence of chlorite degradation activity. Despite the fact that the $\mathrm{p} K_{\mathrm{a}}$ of the alkaline transition is significantly different in the three proteins due to differences in the flexibility of $\mathrm{R} 127$, the $\mathrm{pH}$ dependence of the catalytic efficiency was similar in wild-type CCld and the two variants.

Elucidation of HS and LS complexes of CCld studied at $\mathrm{pH}$ 6.5 demonstrated that addition of a ligand, irrespective of its nature, eliminates W501 but not W502 from the heme cavity and keeps R127 in the "out" conformation. ${ }^{14}$ This also applies for the LS ligand isothiocyanate, which may act as a model for chlorite. ${ }^{14}$ The crystal structure of the CCld-isothiocyanate complex suggests that the substrate is hydrogen bonded to W502 and R127 in the "out" conformation. Immediately after this binding event, chlorite is converted to chloride and dioxygen. It has been demonstrated with $\mathrm{NdCld}$ that in this reaction the distal R173 is important but not fully essential. Exchange of R173 by either alanine or glutamine led to significant decreases in the respective turnover numbers, i.e., $\sim 6.5 \%$ (R173A) and $\sim 5.4 \%$ (R173Q). ${ }^{9}$ In addition to the decreased $k_{\text {cat }}$ values, the $N d$ Cld R173 variants were also more prone to inactivation than the wild-type enzyme by oxidative modification of the heme cofactor and the protein., ${ }^{9,16}$ In any case, the impact of the conformational dynamics of R127 in CCld on the cleavage reaction seems to be small because at the $\mathrm{pH}$ optimum Q74E exhibits the highest turnover number. Apparently, R127 in the arrested "out" conformation is still able to support the homolytic or heterolytic cleavage of chlorite. ${ }^{1,2,6-9,12,14}$

Independently of the mechanism of chlorite cleavage, the resulting transient intermediates (either Compound I and hypochlorite or Compound II and chlorine monoxide) must be kept in the reaction sphere for the following rebound step. It has been demonstrated that the bond cleavage in chlorite occurs very fast and independent of $\mathrm{pH}^{14}$ Thus, it was hypothesized that the following rebound step is responsible for the observed $\mathrm{pH}$ dependence. ${ }^{14}$ In this step, the guanidinium group seems to play an important role by keeping the reaction partners in place. Computational studies suggest that in the rebound step the transient intermediates (either hypochlorite or chlorine monoxide) must rotate. This rotation requires rearrangements of $\mathrm{H}$-bonds with the arginine to support $\mathrm{O}-\mathrm{O}$ bond formation. Finally, the $\mathrm{H}$-bond between the arginine and the two oxygen atoms needs to rearrange again to the terminal chlorine atom. ${ }^{54}$ The data presented here clearly suggest that R127, arrested in a salt bridge with E74 and thus in the "out" conformation, can still participate in these proposed reaction steps.

In addition to the decrease in the rate of chlorite degradation, Clds become inactivated at alkaline $\mathrm{pH}$ and this inactivation reaction is promoted in the absence of the arginine. This is also the case for CCld. ${ }^{13}$ It was proposed that at alkaline $\mathrm{pH}$ values, where $\mathrm{O}-\mathrm{O}$ bond formation apparently becomes inefficient, intermediates (hypochlorite or chlorine monoxide) escape from the reaction sphere, which oxidatively modify both the heme cofactor and the protein. Increasing the level of irreversible inhibition with an increase in $\mathrm{pH}$ is also seen in this study. Here the role of the dynamics of R127 remains elusive because upon exchange of glutamine with either valine or glutamate the extent of inactivation was slightly higher compared to that of the wild-type protein (Figure S7).

In summary, we have demonstrated that the conformational dynamics of the catalytic arginine can be modulated by its remote $\mathrm{H}$-bonding network. This is underlined by the presented high-resolution crystal structures and the impact of the flexibility of R127 on the alkaline transition. Formation of the LS hydroxide complex is shown by UV-vis and EPR spectroscopies, and the calculated $\mathrm{p} K_{\mathrm{a}}$ values strongly depended on the flexibility of R127. By contrast, the effect on the catalytic efficiency in the $\mathrm{pH}$ regime between 5.0 and 9.0 was small, suggesting that R127 in the "out" conformation can support chlorite cleavage and keep the transient intermediates in place. Most interestingly, the strongest impact of the exchange R127 was on the thermal stability and the $K_{\mathrm{M}}$ values at the $\mathrm{pH}$ optimum. This seems to be related to the fact that R127 is H-bonded to the Cld-typical (flexible) $\alpha$-helical loop in CCld that might act as a gatekeeper for the active site. 
Arresting this loop in the salt bridge (Q74E) significantly increases the thermal stability of the heme cavity but at the same time decreases the loop dynamics that is necessary for chlorite to enter the active site and bind to the heme iron.

\section{ASSOCIATED CONTENT}

\section{(s) Supporting Information}

The Supporting Information is available free of charge at https://pubs.acs.org/doi/10.1021/acs.biochem.0c00910.

Multiple-sequence alignments of clade 1 and 2 Clds (Table S1), detailed information about primers (Table S2), EPR simulation parameters (Table S3), and EPR spectra of NdCld, wild-type CCld, and variants Q74V and $\mathrm{Q} 74 \mathrm{E}$ at various $\mathrm{pH}$ values (Figures S3-S5, respectively), data about thermal stabilities at various $\mathrm{pH}$ values (Table S4), electron density maps of Q74V and Q74E (Figure S6), and kinetic parameters (Figure S7) (PDF)

\section{Accession Codes}

Cld from Cyanothece sp. PCC7425 (CCld), UniProt entry B8HNS6, PDB entry 5MAU; Cld from Candidatus "Nitrospira defluvii” (NdCld), UniProt entry B3U4H7, PDB entry 3NN1.

\section{AUTHOR INFORMATION}

\section{Corresponding Authors}

Christian Obinger - Department of Chemistry, Institute of Biochemistry, University of Natural Resources and Life Sciences, Vienna, A-1190 Vienna, Austria; (1) orcid.org/ 0000-0002-7133-3430; Phone: +43-47654-77273; Email: christian.obinger@boku.ac.at; Fax: +43-1-4765477250

Sabine Van Doorslaer - BIMEF Laboratory, Department of Chemistry, University of Antwerp, 2020 Antwerp, Belgium; (1) orcid.org/0000-0002-1685-9202; Phone: +3232652461; Email: sabine.vandoorslaer@uantwerpen.be

\section{Authors}

Daniel Schmidt - Department of Chemistry, Institute of Biochemistry, University of Natural Resources and Life Sciences, Vienna, A-1190 Vienna, Austria

Ilenia Serra - BIMEF Laboratory, Department of Chemistry, University of Antwerp, 2020 Antwerp, Belgium; (1) orcid.org/0000-0003-4537-9635

Georg Mlynek - Department of Structural and Computational Biology, Max Perutz Laboratories, University of Vienna, A-1030 Vienna, Austria

Vera Pfanzagl - Department of Chemistry, Institute of Biochemistry, University of Natural Resources and Life Sciences, Vienna, A-1190 Vienna, Austria; (1) orcid.org/ 0000-0002-9361-8365

Stefan Hofbauer - Department of Chemistry, Institute of Biochemistry, University of Natural Resources and Life Sciences, Vienna, A-1190 Vienna, Austria; 이이.org/ 0000-0003-3375-7715

Paul G. Furtmüller - Department of Chemistry, Institute of Biochemistry, University of Natural Resources and Life Sciences, Vienna, A-1190 Vienna, Austria; 이이.org/ 0000-0002-1199-2469

Kristina Djinovic-Carugo - Department of Structural and Computational Biology, Max Perutz Laboratories, University of Vienna, A-1030 Vienna, Austria; Department of Biochemistry, Faculty of Chemistry and Chemical
Technology, University of Ljubljana, SI-1000 Ljubljana, Slovenia; orcid.org/0000-0003-0252-2972

Complete contact information is available at: https://pubs.acs.org/10.1021/acs.biochem.0c00910

\section{Author Contributions}

${ }^{\perp}$ D.S. and I.S. contributed equally to this work.

\section{Funding}

This paper is part of a project that has received funding from the European Union's Horizon 2020 research and innovation program under Marie Skłodowska-Curie Grant Agreement 813209. D.S. was supported by the Austrian Science Foundation, FWF [Doctoral program BioToP-Molecular Technology of Proteins (W1224) and Projects P30979 and I2429]. Beamtime was granted by the MX682 proposal at EMBL, Hamburg, Germany.

Notes

The authors declare no competing financial interest.

\section{ACKNOWLEDGMENTS}

The DSC equipment was kindly provided by the EQ-BOKU VIBT GmbH and the BOKU Core Facility for Biomolecular \& Cellular Analysis.

\section{ABBREVIATIONS}

Cld, chlorite dismutase; CCld, chlorite dismutase from Cyanothece sp. PCC7425; CW, continuous wave; D, tetragonal; DSC, differential circular calorimetry; E, rhombic; ECD, electronic circular dichroism; EPR, electron paramagnetic resonance; HS, high-spin; LS, low-spin; NdCld, chlorite dismutase from Candidatus "Nitrospira defluvii"; PDB, Protein Data Bank; ZFS, zero-field splitting.

\section{REFERENCES}

(1) Hofbauer, S., Schaffner, I., Furtmüller, P. G., and Obinger, C. (2014) Chlorite dismutases - a heme enzyme family for use in bioremediation and generation of molecular oxygen. Biotechnol. J. 9, 461-473.

(2) Schaffner, I., Hofbauer, S., Krutzler, M., Pirker, K. F., Furtmüller, P. G., and Obinger, C. (2015) Mechanism of chlorite degradation to chloride and dioxygen by the enzyme chlorite dismutase. Arch. Biochem. Biophys. 574, 18-26.

(3) Goblirsch, B., Kurker, R. C., Streit, B. R., Wilmot, C. M., and Dubois, J. L. (2011) Chlorite dismutases, DyPs, and EfeB: 3 microbial heme enzyme families comprise the CDE structural superfamily. $J$. Mol. Biol. 408, 379-398.

(4) Hofbauer, S., Pfanzagl, V., Michlits, H., Schmidt, D., Obinger, C., and Furtmüller, P. G. (2021) Understanding molecular enzymology of porphyrin-binding $\alpha+\beta$ barrel proteins - one fold, multiple functions. Biochim. Biophys. Acta, Proteins Proteomics 1869, 140536.

(5) Ettwig, K. F., Butler, M. K., Le Paslier, D., Pelletier, E., Mangenot, S., Kuypers, M. M. M., Schreiber, F., Dutilh, B. E., Zedelius, J., De Beer, D., Gloerich, J., Wessels, H. J. C. T., Van Alen, T., Luesken, F., Wu, M. L., Van De Pas-Schoonen, K. T., Op Den Camp, H. J. M., Janssen-Megens, E. M., Francoijs, K. J., Stunnenberg, H., Weissenbach, J., Jetten, M. S. M., and Strous, M. (2010) Nitritedriven anaerobic methane oxidation by oxygenic bacteria. Nature 464, $543-548$

(6) de Geus, D. C., Thomassen, E. A. J., Hagedoorn, P. L., Pannu, N. S., van Duijn, E., and Abrahams, J. P. (2009) Crystal Structure of chlorite dismutase, a detoxifying enzyme producing molecular oxygen. J. Mol. Biol. 387, 192-206.

(7) Goblirsch, B. R., Streit, B. R., DuBois, J. L., and Wilmot, C. M. (2010) Structural features promoting dioxygen production by 
Dechloromonas aromatica chlorite dismutase. JBIC, J. Biol. Inorg. Chem. $15,879-888$.

(8) Kostan, J., Sjöblom, B., Maixner, F., Mlynek, G., Furtmüller, P. G., Obinger, C., Wagner, M., Daims, H., and Djinović-Carugo, K. (2010) Structural and functional characterisation of the chlorite dismutase from the nitrite-oxidizing bacterium "Candidatus Nitrospira defluvii": Identification of a catalytically important amino acid residue. J. Struct. Biol. 172, 331-342.

(9) Hofbauer, S., Gysel, K., Bellei, M., Hagmüller, A., Schaffner, I., Mlynek, G., Kostan, J., Pirker, K. F., Daims, H., Furtmüller, P. G., Battistuzzi, G., Djinović-Carugo, K., and Obinger, C. (2014) Manipulating conserved heme cavity residues of chlorite dismutase: effect on structure, redox chemistry, and reactivity. Biochemistry 53, 77-89.

(10) Freire, D. M., Rivas, M. G., Dias, A. M., Lopes, A. T., Costa, C., Santos-Silva, T., Van Doorslaer, S., and González, P. J. (2015) The homopentameric chlorite dismutase from Magnetospirillum sp. J. Inorg. Biochem. 151, 1-9.

(11) Mlynek, G., Sjöblom, B., Kostan, J., Füreder, S., Maixner, F., Gysel, K., Furtmüller, P. G., Obinger, C., Wagner, M., Daims, H., and Djinovic-Carugo, K. (2011) Unexpected diversity of chlorite dismutases: A catalytically efficient dimeric enzyme from Nitrobacter winogradskyi. J. Bacteriol. 193, 2408-2417.

(12) Celis, A. I., Geeraerts, Z., Ngmenterebo, D., Machovina, M. M., Kurker, R. C., Rajakumar, K., Ivancich, A., Rodgers, K. R., LukatRodgers, G. S., and Dubois, J. L. (2015) A dimeric chlorite dismutase exhibits $\mathrm{O}_{2}$-generating activity and acts as a chlorite antioxidant in Klebsiella pneumoniae MGH 78578. Biochemistry 54, 434-446.

(13) Schaffner, I., Hofbauer, S., Krutzler, M., Pirker, K. F., Bellei, M., Stadlmayr, G., Mlynek, G., Djinovic-Carugo, K., Battistuzzi, G., Furtmüller, P. G., Daims, H., and Obinger, C. (2015) Dimeric chlorite dismutase from the nitrogen-fixing cyanobacterium Cyanothece sp. PCC7425. Mol. Microbiol. 96, 1053-1068.

(14) Schaffner, I., Mlynek, G., Flego, N., Pühringer, D., LibisellerEgger, J., Coates, L., Hofbauer, S., Bellei, M., Furtmüller, P. G., Battistuzzi, G., Smulevich, G., Djinović-Carugo, K., and Obinger, C. (2017) Molecular mechanism of enzymatic chlorite detoxification: Insights from structural and kinetic studies. ACS Catal. 7, 7962-7976.

(15) Hofbauer, S., Bellei, M., Sündermann, A., Pirker, K. F., Hagmüller, A., Mlynek, G., Kostan, J., Daims, H., Furtmüller, P. G., Djinović-Carugo, K., Oostenbrink, C., Battistuzzi, G., and Obinger, C. (2012) Redox thermodynamics of high-spin and low-spin forms of chlorite dismutases with diverse subunit and oligomeric structures. Biochemistry 51, 9501-9512.

(16) Hofbauer, S., Gruber, C., Pirker, K. F., Sündermann, A., Schaffner, I., Jakopitsch, C., Oostenbrink, C., Furtmüller, P. G., and Obinger, C. (2014) Transiently produced hypochlorite is responsible for the irreversible inhibition of chlorite dismutase. Biochemistry 53, 3145-3157.

(17) Blanc, B., Mayfield, J. A., McDonald, C. A., Lukat-Rodgers, G. S., Rodgers, K. R., and DuBois, J. L. (2012) Understanding how the distal environment directs reactivity in chlorite dismutase: spectroscopy and reactivity of Arg183 mutants. Biochemistry 51, 1895-1910.

(18) Streit, B. R., Blanc, B., Lukat-Rodgers, G. S., Rodgers, K. R., and DuBois, J. L. (2010) How active-site protonation state influences the reactivity and ligation of the heme in chlorite dismutase. J. Am. Chem. Soc. 132, 5711-5724.

(19) Thompson, J. D., Higgins, D. G., and Gibson, T. J. (1994) CLUSTAL W: Improving the sensitivity of progressive multiple sequence alignment through sequence weighting, position-specific gap penalties and weight matrix choice. Nucleic Acids Res. 22, 4673-4680.

(20) Kumar, S., Stecher, G., Li, M., Knyaz, C., and Tamura, K. (2018) MEGA X: Molecular evolutionary genetics analysis across computing platforms. Mol. Biol. Evol. 35, 1547-1549.

(21) Le Breton, N., Wright, J. J., Jones, A. J.Y., Salvadori, E., Bridges, H. R., Hirst, J., and Roessler, M. M. (2017) Using Hyperfine Electron Paramagnetic Resonance Spectroscopy to Define the Proton-Coupled Electron Transfer Reaction at Fe-S Cluster N2 in Respiratory Complex I. J. Am. Chem. Soc. 139, 16319-16326.
(22) Stoll, S., and Schweiger, A. (2006) EasySpin, a comprehensive software package for spectral simulation and analysis in EPR. J. Magn. Reson. 178, 42-55.

(23) Cianci, M., Bourenkov, G., Pompidor, G., Karpics, I., Kallio, J., Bento, I., Roessle, M., Cipriani, F., Fiedler, S., and Schneider, T. R. (2017) P13, the EMBL macromolecular crystallography beamline at the low-emittance PETRA III ring for high- and low-energy phasing with variable beam focusing. J. Synchrotron Radiat. 24, 323-332.

(24) Kabsch, W. (2010) XDS. Acta Crystallogr., Sect. D: Biol. Crystallogr. 66, 125-132.

(25) Karplus, P. A., and Diederichs, K. (2012) Linking crystallographic model and data quality. Science 336, 1030-1033.

(26) McCoy, A. J., Grosse-Kunstleve, R. W., Adams, P. D., Winn, M. D., Storoni, L. C., and Read, R. J. (2007) Phaser crystallographic software. J. Appl. Crystallogr. 40, 658-674.

(27) Emsley, P., Lohkamp, B., Scott, W. G., and Cowtan, K. (2010) Features and development of Coot. Acta Crystallogr., Sect. D: Biol. Crystallogr. 66, 486-501.

(28) Adams, P. D., Afonine, P. V., Bunkóczi, G., Chen, V. B., Davis, I. W., Echols, N., Headd, J. J., Hung, L. W., Kapral, G. J., GrosseKunstleve, R. W., McCoy, A. J., Moriarty, N. W., Oeffner, R., Read, R. J., Richardson, D. C., Richardson, J. S., Terwilliger, T. C., and Zwart, P. H. (2010) PHENIX: A comprehensive Python-based system for macromolecular structure solution. Acta Crystallogr., Sect. D: Biol. Crystallogr. 66, 213-221.

(29) French, S., and Wilson, K. (1978) On the treatment of negative intensity observations. Acta Crystallogr., Sect. A: Cryst. Phys., Diffr., Theor. Gen. Crystallogr. 34, 517-525.

(30) Merritt, E. A. (2012) To B or not to B: A question of resolution? Acta Crystallogr., Sect. D: Biol. Crystallogr. 68, 468-477.

(31) Bacchi, A., Lamzin, V. S., and Wilson, K. S. (1996) A selfvalidation technique for protein structure refinement: The extended Hamilton test. Acta Crystallogr., Sect. D: Biol. Crystallogr. 52, 641-646.

(32) Hamilton, W. C. (1965) Significance tests on the crystallographic R factor. Acta Crystallogr. 18, 502-510.

(33) Joosten, R. P., Salzemann, J., Bloch, V., Stockinger, H., Berglund, A. C., Blanchet, C., Bongcam-Rudloff, E., Combet, C., Da Costa, A. L., Deleage, G., Diarena, M., Fabbretti, R., Fettahi, G., Flegel, V., Gisel, A., Kasam, V., Kervinen, T., Korpelainen, E., Mattila, K., Pagni, M., Reichstadt, M., Breton, V., Tickle, I. J., and Vriend, G. (2009) PDB-REDO: Automated re-refinement of X-ray structure models in the PDB. J. Appl. Crystallogr. 42, 376-384.

(34) Davis, I. W., Murray, L. W., Richardson, J. S., and Richardson, D. C. (2004) MOLPROBITY: structure validation and all-atom contact analysis for nucleic acids and their complexes. Nucleic Acids Res. 32, W615-W619.

(35) Jakopitsch, C., Pirker, K. F., Flemmig, J., Hofbauer, S., Schlorke, D., Furtmüller, P. G., Arnhold, J., and Obinger, C. (2014) Mechanism of reaction of chlorite with mammalian heme peroxidases. J. Inorg. Biochem. 135, 10-19.

(36) Furman, C. S., and Margerum, D. W. (1998) Mechanism of chlorine dioxide and chlorate Ion formation from the reaction of hypobromous acid and chlorite Ion. Inorg. Chem. 37, 4321-4327.

(37) Telser, J. (2018) EPR Interactions - Zero-field Splittings. In EPR Spectroscopy Fundamentals and Methods (Goldfarb, D., and Stoll, S., Eds.) 1st ed., pp 29-62, John Wiley \& Sons Ltd.

(38) De Schutter, A., Correia, H. D., Freire, D. M., Rivas, M. G., Rizzi, A., Santos-Silva, T., González, P. J., and Van Doorslaer, S. (2015) Ligand binding to chlorite dismutase from Magnetospirillum sp. J. Phys. Chem. B 119, 13859-13869.

(39) Hagedoorn, P. L., De Geus, D. C., and Hagen, W. R. (2002) Spectroscopic characterization and ligand-binding properties of chlorite dismutase from the chlorate respiring bacterial strain GR-1. Eur. J. Biochem. 269, 4905-4911.

(40) Stenklo, K., Danielsson Thorell, H., Bergius, H., Aasa, R., and Nilsson, T. (2001) Chlorite dismutase from Ideonella dechloratans. JBIC, J. Biol. Inorg. Chem. 6, 601-607.

(41) Hofbauer, S., Gysel, K., Mlynek, G., Kostan, J., Hagmüller, A., Daims, H., Furtmüller, P. G., Djinović-Carugo, K., and Obinger, C. 
(2012) Impact of subunit and oligomeric structure on the thermal and conformational stability of chlorite dismutases. Biochim. Biophys. Acta, Proteins Proteomics 1824, 1031-1038.

(42) Yonetani, T., Wilson, D. F., and Seamonds, B. (1966) Studies on cytochrome $c$ peroxidase. J. Biol. Chem. 241, 5347-5352.

(43) Yonetani, T., and Anni, H. (1987) Yeast cytochrome $c$ peroxidase. Coordination and spin states of heme prosthetic group. J. Biol. Chem. 262, 9547-9554.

(44) Celis, A. I., Geeraerts, Z., Ngmenterebo, D., Machovina, M. M., Kurker, R. C., Rajakumar, K., Ivancich, A., Rodgers, K. R., LukatRodgers, G. S., and Dubois, J. L. (2015) A dimeric chlorite dismutase exhibits $\mathrm{O}_{2}$-generating activity and acts as a chlorite antioxidant in Klebsiella pneumoniae MGH 78578. Biochemistry 54, 434-446.

(45) Wittenberg, B. A., Kampa, L., Wittenberg, J. B., Blumberg, W. E., and Peisach, J. (1968) The electronic structure of protoheme proteins. An electron paramagnetic resonance and optical study of cytochrome $c$ peroxidase and its derivatives. J. Biol. Chem. 243, 18631870.

(46) Svistunenko, D. A., Sharpe, M. A., Nicholls, P., Blenkinsop, C., Davies, N. A., Dunne, J., Wilson, M. T., and Cooper, C. E. (2000) The $\mathrm{pH}$ dependence of naturally occurring low-spin forms of methaemoglobin and metmyoglobin: An EPR study. Biochem. J. $351,595-605$.

(47) Tilleman, L., Germani, F., De Henau, S., Helbo, S., Desmet, F., Berghmans, H., Van Doorslaer, S., Hoogewijs, D., Schoofs, L., Braeckman, B. P., Moens, L., Fago, A., and Dewilde, S. (2015) A globin domain in a neuronal transmembrane receptor of Caenorhabditis elegans and Ascaris suum: molecular modeling and functional properties. J. Biol. Chem. 290, 10336-10352.

(48) Nicoletti, F. P., Droghetti, E., Howes, B. D., Bustamante, J. P., Bonamore, A., Sciamanna, N., Estrin, D. A., Feis, A., Boffi, A., and Smulevich, G. (2013) H-bonding networks of the distal residues and water molecules in the active site of Thermobifida fusca hemoglobin. Biochim. Biophys. Acta, Proteins Proteomics 1834, 1901-1909.

(49) Blumberg, W. E. (1981) The study of hemoglobin by electron paramagnetic resonance spectroscopy. Methods Enzymol. 76, 312329.

(50) Taylor, C. P. S. (1977) The EPR of low spin heme complexes Relation of the $\tau 2 \mathrm{~g}$ hole model to the directional properties of the $\mathrm{g}$ tensor, and a new method for calculating the ligand field parameters. Biochim. Biophys. Acta, Protein Struct. 491, 137-148.

(51) Danielsson Thorell, H., Beyer, N. H., Heegaard, N. H. H., Öhman, M., and Nilsson, T. (2004) Comparison of native and recombinant chlorite dismutase from Ideonella dechloratans. Eur. J. Biochem. 271, 3539-3546.

(52) Van Doorslaer, S., van den Bosch, M., Tilleman, L., and Dewilde, S. (2015) EPR Analysis of imidazole binding to Methanosarcina acetivorans protoglobin. Appl. Magn. Reson. 46, 421433.

(53) Sündermann, A., Reif, M. M., Hofbauer, S., Obinger, C., and Oostenbrink, C. (2014) Investigation of ion binding in chlorite dismutases by means of molecular dynamics simulations. Biochemistry $53,4869-4879$.

(54) Sun, S., Li, Z. S., and Chen, S. L. (2014) A dominant homolytic $\mathrm{O}-\mathrm{Cl}$ bond cleavage with low-spin triplet-state $\mathrm{Fe}(\mathrm{IV})=\mathrm{O}$ formed is revealed in the mechanism of heme-dependent chlorite dismutase. Dalton Trans. 43, 973-981. 